\title{
Effects of anharmonic strain on phase stability of epitaxial films and superlattices: applications to noble metals
}

\author{
V. Ozoliņš, C. Wolverton, and Alex Zunger \\ National Renewable Energy Laboratory, Golden, CO 80401
}

(September 12, 1997)

\begin{abstract}
Epitaxial strain energies of epitaxial films and bulk superlattices are studied via first-principles total energy calculations using the local-density approximation. Anharmonic effects due to large lattice mismatch, beyond the reach of the harmonic elasticity theory, are found to be very important in $\mathrm{Cu} / \mathrm{Au}$ (lattice mismatch $12 \%), \mathrm{Cu} / \mathrm{Ag}(12 \%)$ and $\mathrm{Ni} / \mathrm{Au}(15 \%)$. We find that $\langle 001\rangle$ is the elastically soft direction for biaxial expansion of $\mathrm{Cu}$ and $\mathrm{Ni}$, but it is $\langle 201\rangle$ for large biaxial compression of $\mathrm{Cu}$, $\mathrm{Ag}$, and $\mathrm{Au}$. The stability of superlattices is discussed in terms of the coherency strain and interfacial energies. We find that in phase-separating systems such as $\mathrm{Cu}-\mathrm{Ag}$ the superlattice formation energies decrease with superlattice period, and the interfacial energy is positive. Superlattices are formed easiest on (001) and hardest on (111) substrates. For ordering systems, such as $\mathrm{Cu}-\mathrm{Au}$ and $\mathrm{Ag}-\mathrm{Au}$, the formation energy of superlattices increases with period, and interfacial energies are negative. These superlattices are formed easiest on (001) or (110) and hardest on (111) substrates. For Ni$\mathrm{Au}$ we find a hybrid behavior: superlattices along $\langle 111\rangle$ and $\langle 001\rangle$ behave like in phase-separating systems, while for $\langle 110\rangle$ they behave like in ordering systems. Finally, recent experimental results on epitaxial stabilization of disordered $\mathrm{Ni}-\mathrm{Au}$ and $\mathrm{Cu}-\mathrm{Ag}$ alloys, immiscible in the bulk form, are explained in terms of destabilization of the phase separated state due to lattice mismatch between the substrate and constituents.
\end{abstract}

PACS numbers: 62.20.Dc, 68.60.-p, 81.10.Aj

\section{INTRODUCTION}

Recently, there has been much interest ${ }^{1-14}$ in growth of epitaxial metal films and superlattices due to their unusual physical properties. The quality and structure of these systems is of paramount importance for applications. Epitaxial monolayer and multilayer (up to 10 layers) formation has been observed for many metal/semiconductor and metal/metal combinations. Most metal/metal superlattices have been grown for elements in different crystal structures (e.g., fcc/bcc) and with considerable size mismatch (e.g., $10 \%$ for $\mathrm{Cu} / \mathrm{Nb}^{15^{-17}}$ ). Furthermore, elemental metals and alloys have been found to form epitaxially in structures which are unstable in bulk form. ${ }^{18-22}$ Recently, the topic of surface alloy formation in bulk immiscible systems has attracted considerable attention. ${ }^{23-42}$ These systems are usually strained due to film/substrate lattice mismatch. One would like to understand and predict the stability of these types of strained materials. In order to do so, one requires knowledge of two types of energies. The stability of epitaxial $A_{1-x} B_{x}$ alloy films and strained $A_{p} B_{q}$ superlattices depends on (i) the energies of coherently strained constituents $A$ and $B$, and (ii) the formation energy of $A_{1-x} B_{x}$ or $A_{p} B_{q}$ itself. Regarding (i), previous theoretical studies ${ }^{18,43-56}$ have described these energies using harmonic models, but we are interested here in large strains for which the harmonic theory could break down. Thus, we develop a generalization of previous methods to treat the anharmonic epitaxial strain energies of the con- stituents. Regarding (ii), these energies depend on the configuration degrees of freedom of the epitaxial film, so their calculation requires statistical methods. ${ }^{56,57}$ In the present paper we investigate items (i) and (ii) above using accurate first-principles LDA calculations.

As for (i), the constituent strain energy, we find that the harmonic strain theory ${ }^{18,46}$ predicting a single, universal relation for elastically soft directions, breaks down for sufficiently large substrate/film lattice mismatch. We find that under biaxial expansion, noble metals are soft along $\langle 001\rangle$, but that under compression the soft direction changes to $\langle 201\rangle$. It is shown that the softness of $\langle 001\rangle$ is a consequence of low bcc/fcc energy differences in noble metals, while the softness of $\langle 201\rangle$ under compressive strain can be explained by loose packing of atoms in the $\{201\}$ planes. Furthermore, the elastic strain energy as a function of direction exhibits qualitative shifts in the hard and soft strain directions, which cannot be guessed from the harmonic elasticity theory. For instance, we find that $\langle 110\rangle$ becomes the hardest direction under biaxial expansion, and $\langle 201\rangle$ becomes the softest direction under biaxial compression, while the harmonic theory always predicts either $\langle 111\rangle$ as the hardest and $\langle 001\rangle$ as the softest direction, or vice versa.

Regarding (ii), the formation energy, we find that the anomalous elastic softness of the constituents along $\langle 001\rangle$ and $\langle 201\rangle$ leads to low constituent strain energy in superlattices along these directions, which makes them more stable than superlattices along other $\widehat{G}$. For instance, in the size-mismatched systems $\mathrm{Cu}-\mathrm{Au}, \mathrm{Cu}-\mathrm{Ag}$, and $\mathrm{Ni}-\mathrm{Au}$, 
$A_{n} B_{n}$ superlattices along $\langle 001\rangle$ are the most stable for all periods $n$. Interfacial energies are found to be negative in $\mathrm{Ag}-\mathrm{Au}$ and $\mathrm{Cu}-\mathrm{Au}$ (reflecting their bulk miscibility), and positive in the phase separating systems $\mathrm{Cu}-\mathrm{Ag}$ and $\mathrm{Ni}-\mathrm{Au}$. However, attraction between (110) interfaces in $\mathrm{Ni}-\mathrm{Au}$ is very strong and favors short-period $(n \propto 2)$ superlattices over long-period superlattices with few interfaces.

In the case of epitaxially grown disordered alloys, we find that the biaxial constraint on the phase separated constituents may stabilize the alloy with respect to phase separation. The stabilization effect is always greater on substrates oriented along elastically hard directions (i.e., with high constituent strain energy) like $\langle 111\rangle$ than along soft directions like $\langle 001\rangle$. For instance, on latticematched substrates, epitaxial $\mathrm{Ni}_{0.5} \mathrm{Au}_{0.5}$ alloys are stable at all temperatures, and $\mathrm{Cu}_{0.5} \mathrm{Ag}_{0.5}$ alloys are stable for $T>150 \mathrm{~K}$ if grown on a (111) substrate, although both these systems phase separate in bulk form or if grown on a (001) substrate. These predictions agree very well with recent experimental observations. ${ }^{31,36}$

\section{BULK AND EPITAXIAL STABILITY CRITERIA}

The stability of either free-standing or coherently strained alloys and superlattices requires specification of (i) epitaxial strain energies of pure constituents due to film/substrate lattice mismatch, (ii) formation enthalpies of disordered alloys (with respect to either strained or unstrained bulk constituents) and superlattices. In this section, we define these quantities and discuss the physical situations where they should be used.

\section{A. Epitaxial strain energies of elemental constituents}

We start by considering (i) above, which is a common element to alloys and superlattices. Consider a film of pure element $A$ coherently strained on a substrate oriented along direction $\widehat{G}$ with surface unit cell vectors $\mathbf{a}_{1}$ and $\mathbf{a}_{2}$, orthogonal to $\widehat{G}$. We assume that the film, being much thinner than the substrate, maintains coherency with the substrate and plastically deforms to accomodate the lattice mismatch at the interface. This assumption is valid for films thinner than the critical thickness for the nucleation of misfit dislocations. Furthermore, we consider films which are thick enough so that the chemical interaction energy at the film/substrate interface and film/vacuum surface is negligibly small in comparison with the elastic deformation energy of the film. Under these assumptions, the epitaxial strain energy $\Delta E_{A}^{\mathrm{epi}}\left(\mathbf{a}_{1}, \mathbf{a}_{2}, \widehat{G}\right)$ of film $A$ is the strain energy of element $A$ deformed in the growth plane to the unit cell vectors $\left\{\mathbf{a}_{1}, \mathbf{a}_{2}\right\}$ of the substrate, and relaxed with respect to the out-of-plane vector $\mathbf{c}$ :
$\Delta E_{A}^{\mathrm{epi}}\left(\mathbf{a}_{1}, \mathbf{a}_{2}, \widehat{G}\right)=\min _{\mathbf{c}}\left[E_{A}^{\mathrm{tot}}\left(\mathbf{a}_{1}, \mathbf{a}_{2}, \mathbf{c}\right)\right]-E_{A}^{\mathrm{tot}}\left(a_{A}\right)$.

In what follows, we are interested in the case where both the substrate and the unstrained bulk element $A$ have the fcc crystal lattice. Then $\mathbf{a}_{1}$ and $\mathbf{a}_{2}$ are proportional to the equilibrium unstrained lattice vectors of fcc $A, \mathbf{a}) 0_{i}(A)$ :

$$
\mathbf{a}_{i}=\left(\frac{a_{s}}{a_{A}}\right) \mathbf{a}_{i}^{0}(A), \quad i=1,2,
$$

where $a_{s}$ and $a_{A}$ are fcc lattice parameters of the substrate and $A$, correspondingly. The epitaxial strain energy becomes a function of the substrate lattice constant and direction $\widehat{G}$ only:

$$
\Delta E_{A}^{\mathrm{epi}}\left[\left(a_{s} / a_{A}\right) \mathbf{a}_{1},\left(a_{s} / a_{A}\right) \mathbf{a}_{2}, \widehat{G}\right] \equiv \Delta E_{A}^{\mathrm{epi}}\left(a_{s}, \widehat{G}\right) .
$$

LDA calculations of $\Delta E_{A}^{\mathrm{epi}}\left(a_{s}, \widehat{G}\right)$ are described in Sec. III.

\section{B. Formation enthalpies of alloys and superlattices}

Like the formation enthalpy of any ordered bulk compound, the formation enthalpy $\Delta H_{\mathrm{SL}}^{\mathrm{bulk}}(p q, \widehat{G})$ of an $A_{p} B_{q}$ unstrained (bulk) superlattice is defined as the energy gain or loss with respect to unstrained bulk constituents:

$$
\begin{aligned}
& \Delta H_{\mathrm{SL}}^{\mathrm{bulk}}(p q, \widehat{G})=E^{\mathrm{tot}}\left(A_{p} B_{q}, \widehat{G}\right) \\
& -\left[\frac{p}{p+q} E_{A}^{\mathrm{tot}}\left(a_{A}\right)+\frac{q}{p+q} E_{B}^{\mathrm{tot}}\left(a_{B}\right)\right],
\end{aligned}
$$

where $a_{A}$ is the equilibrium lattice constant of the unstrained bulk element $A$ and $E_{A}^{\text {tot }}\left(a_{A}\right)$ is the total energy of $A$. This enthalpy characterizes the propensity to form superlattices with respect to the phase separated bulk constituents. If $\Delta H_{\mathrm{SL}}^{\text {bulk }}(p q, \widehat{G})<0$, the unstrained superlattices are energetically favored over the phase separation, while the phase separated state is favored if $\Delta H_{\mathrm{SL}}^{\text {bulk }}(p q, \widehat{G})>0$. To be stable, freestanding bulk superlattices must satisfy stability criteria with respect to at least: (i) phase separation into unstrained bulk constituents and (ii) formation of a configurationally disordered bulk alloy. The bulk mixing enthalpy, $\Delta H_{\text {mix }}^{\text {bulk }}\left(A_{1-x} B_{x}\right)$, of the alloy is given by:

$$
\begin{aligned}
& \Delta H_{\text {mix }}^{\text {bulk }}\left(A_{1-x} B_{x}\right)=E^{\text {tot }}\left(A_{1-x} B_{x}\right) \\
& -\left[(1-x) E_{A}^{\text {tot }}\left(a_{A}\right)+x E_{B}^{\text {tot }}\left(a_{B}\right)\right],
\end{aligned}
$$

where $x=q /(p+q)$ is the composition and $E^{\text {tot }}\left(A_{1-x} B_{x}\right)$ is the total energy per atom of the configurationally random alloy.

If $\Delta H_{\mathrm{mix}}^{\text {bulk }}\left(A_{1-x} B_{x}\right)<\Delta H_{\mathrm{SL}}^{\mathrm{bulk}}\left(A_{p} B_{q}\right)<0$, then both the superlattice and disordered alloy are stable with respect to phase separation, but the superlattice is unstable with respect to disordering. However, if 
$\Delta H_{\mathrm{SL}}^{\text {bulk }}\left(A_{p} B_{q}\right)<\Delta H_{\text {mix }}^{\text {bulk }}\left(A_{1-x} B_{x}\right)<0$, then superlattices are stable with respect to both phase separation and disordering, and it may be possible to grow them.

The bulk formation enthalpy of a superlattice, $\Delta H_{\mathrm{SL}}^{\text {bulk }}(p q, \widehat{G})$, can be separated into two components. To identify them, it is useful to first consider the infinite period superlattice limit $p, q \rightarrow \infty$, where $A / B$ interfacial interactions contribute a negligible amount of order $\mathcal{O}(1 / p)$. In this case, the bulk formation enthalpy of $A_{\infty} B_{\infty}$ superlattice is given by

$$
\begin{aligned}
& \Delta H_{\mathrm{SL}}^{\mathrm{bulk}}(p q \rightarrow \infty, \widehat{G}) \equiv \Delta E_{\mathrm{CS}}^{\mathrm{eq}}(x, \widehat{G}) \\
& =\min _{\mathbf{a}_{1}, \mathbf{a}_{2}}\left[(1-x) \Delta E_{A}^{\mathrm{epi}}\left(\mathbf{a}_{1}, \mathbf{a}_{2}, \widehat{G}\right)+x \Delta E_{B}^{\mathrm{epi}}\left(\mathbf{a}_{1}, \mathbf{a}_{2}, \widehat{G}\right)\right],
\end{aligned}
$$

where $\Delta E_{A}^{\text {epi }}$ is the epitaxial deformation energy of $A$, given by Eq. (1). We define this energy as the "constituent strain" (CS) to emphasize that in this limit the superlattice formation enthalpy depends only on its strained constituents. This is also the energy required to keep $A$ and $B$ coherent.

For finite-period superlattices, the formation energy is determined not only by the elastic strain energy, but also by interactions between unlike atoms at $A / B$ interfaces. We define this interfacial energy $I(p q, \widehat{G})$ as:

$$
\Delta H_{\mathrm{SL}}^{\mathrm{bulk}}(p q, \widehat{G})-\Delta H_{\mathrm{SL}}^{\mathrm{bulk}}(p q \rightarrow \infty, \widehat{G}) \equiv \frac{4}{p+q} I(p q, \widehat{G}) .
$$

It is the total energy per layer of a single interface between infinite slabs of $A$ and $B$ oriented along $\widehat{G}$. $I(\infty)<0$ signals that the interface is energetically favored, while $I(\infty)>0$ indicates that an isolated interface is not preferred, and long-period superlattices with fewer interfaces are usually more stable than the short-period ones (however, this simple argument is not always true, see the following discussion).

For equiatomic $(A)_{n} /(B)_{n}$ superlattices Eq. (7) becomes:

$$
\Delta H_{\mathrm{SL}}^{\mathrm{bulk}}(n, \widehat{G})=\frac{2 I(n, \widehat{G})}{n}+\Delta E_{\mathrm{CS}}^{\mathrm{eq}}(x=0.5, \widehat{G}) .
$$

For small $n$ interfaces will interact with each other. We describe this process by the interface interaction energy $\delta I(n, \widehat{G})$ :

$$
\delta I(n, \widehat{G})=I(n, \widehat{G})-I(n \rightarrow \infty, \widehat{G}) .
$$

Negative $\delta I(n, \widehat{G})$ may favor short-period superlattices over long-period superlattices even if the interfacial en$\operatorname{ergy} I(n \rightarrow \infty, \widehat{G})$ is positive. For this to happen it is necessary that

$$
\delta I(n, \widehat{G})<-|I(n \rightarrow \infty, \widehat{G})|
$$

In Sec. IV C we show that this unusual phenomenon occurs in $\mathrm{Ni}-\mathrm{Au}$.
If a disordered alloy is grown epitaxially on a latticematched fcc substrate, its stability with respect to phase separation is given by the epitaxial mixing enthalpy:

$$
\begin{aligned}
& \delta H_{\text {mix }}^{\text {epi }}\left(A_{1-x} B_{x}\right)=\Delta H_{\text {mix }}^{\text {bulk }}\left(A_{1-x} B_{x}\right) \\
& -(1-x) \Delta E_{A}^{\mathrm{epi}}\left(a_{s}, \widehat{G}\right)-x \Delta E_{B}^{\mathrm{epi}}\left(a_{s}, \widehat{G}\right),
\end{aligned}
$$

where $\Delta E_{A}^{\text {epi }}\left(a_{s}, \widehat{G}\right)$ is the epitaxial strain energy of Eq. (3), accounting for the fact that the phase-separated consituents must also be lattice-matched with the substrate. Due to the presence of these terms, disordered alloys may form epitaxially $\left[\delta H_{\text {mix }}^{\mathrm{epi}}\left(A_{1-x} B_{x}\right)<0\right]$ even if the corresponding bulk alloys phase separate $\left[\Delta H_{\text {mix }}^{\text {bulk }}\left(A_{1-x} B_{x}\right)>0\right]$. This situation is especially likely to occur for elastically hard directions $\widehat{G}$ with large values of $\Delta E_{A, B}^{\mathrm{epi}}\left(a_{s}, \widehat{G}\right)$, for instance $\langle 111\rangle$ and $\langle 110\rangle$ (see Sec III B).

The objective of this work is to calculate $\Delta H_{\mathrm{SL}}^{\text {bulk }}\left(A_{p} B_{q}\right)$ [Eq. (4)], $\Delta H_{\text {mix }}^{\text {bulk }}\left(A_{1-x} B_{x}\right)$ [Eq. (5)] and $\delta H_{\text {mix }}^{\text {epi }}\left(A_{1-x} B_{x}\right)$ [Eq. (11)] from first principles for $\mathrm{Ag}-\mathrm{Au}, \mathrm{Cu}-\mathrm{Ag}, \mathrm{Cu}-\mathrm{Au}$, and $\mathrm{Ni}-\mathrm{Au}$. This requires:

(a) Epitaxial strain energies of pure constituents, $\Delta E_{A}^{\text {epi }}\left(a_{s}, \widehat{G}\right)$ [Eq. (3)], for $\mathrm{Ag}, \mathrm{Au}, \mathrm{Cu}$ and Ni. This is described in Sec. III.

(b) Equilibrium constituent strain energy $\Delta E_{\mathrm{CS}}^{\mathrm{eq}}$ [Eq. (6)] for $\mathrm{Ag}-\mathrm{Au}, \mathrm{Cu}-\mathrm{Ag}, \mathrm{Cu}-\mathrm{Au}$, and $\mathrm{Ni}-\mathrm{Au}$. This is described in Sec. IV A.

(c) The interfacial energy $I(p q, \widehat{G})$ of Eq. (8) requires $\Delta H_{\text {form }}^{\text {bulk }}\left(A_{p} B_{q}, \widehat{G}\right)$ for arbitrary $p q$ and $\widehat{G}$. $\Delta H_{\text {mix }}^{\text {bulk }}\left(A_{1-x} B_{x}\right)$ and $\delta H_{\text {mix }}^{\text {epi }}\left(A_{1-x} B_{x}\right)$ require the total energy of a configurationally disordered solid solution. All these quantities are obtained from the mixed-space cluster expansion as described in Sec. IV B.

\section{ELEMENTAL EPITAXIAL FILMS}

\section{A. Anharmonic epitaxial strain in thin films of pure elements: Analytic forms}

The epitaxial strain energy [Eq. (3)] of a film of element $A$ (with an equilibrium fcc lattice constant $a_{A}$ ) on a fcc substrate with lattice constant $a_{s}$, oriented along direction $\widehat{G}$, is conveniently obtained in a two-step process considered by Hornstra and Bartels. ${ }^{43}$ First, the fcc crystal of bulk $A$ is uniformly stretched (or compressed) to the lattice constant of the substrate $a_{s}$. The energy change relative to free $A$ is given by the hydrostatic bulk deformation energy $\Delta E_{A}^{\text {bulk }}\left(a_{s}\right)$. In the second step, outof-plane unit cell vector $\mathbf{c}$ of the film relaxes to satisfy Eq. (1). The change $\Delta \mathbf{c}=\mathbf{c}-\left(a_{s} / a_{A}\right) \mathbf{c}^{0}$ (where $\mathbf{c}^{0}$ is the fcc lattice vector of unstrained $A$ ), has components parallel $\left[\Delta \mathbf{c}_{\|}\right]$and perpendicular $\left[\Delta \mathbf{c}_{\perp}\right]$ to the growth direction $\widehat{G}$. The parallel component $\Delta \mathbf{c}_{\|}$changes the volume of the unit cell and thus has a large effect on the total energy. In contrast, the so-called shear strain $\Delta c_{\perp}$ shifts 
planes orthogonal to $\widehat{G}$ and does not change the volume of the unit cell. Consequently, it has a much smaller effect on the total energy. Furthermore, this strain vanishes by symmetry for directions $\langle 001\rangle,\langle 111\rangle$ and $\langle 110\rangle$, and the shear strain energy must have zero angular derivatives at these points. Therefore, we neglect the shear strain $\Delta c_{\perp}$ also for low-symmetry directions. Bottomley and Fons ${ }^{46}$ have shown that this approximation introduces rather small errors in the harmonic epitaxial strain energies.

Neglecting the shear strain $\Delta \mathbf{c}_{\perp}$, the strain energy of element $A$ is then a function of the direction $\widehat{G}$ and two scalar variables, $a_{s}$ and $\epsilon_{\|}=\left|\Delta \mathbf{c}_{\|}\right| / a_{s}-1$. The epitaxial strain energy $\Delta E_{A}^{\mathrm{epi}}\left(a_{s}, \widehat{G}\right)$ of Eq. (3) is the minimum of the strain energy with respect to $\epsilon_{\|}$at a fixed substrate lattice constant $a_{s}$ :

$$
\Delta E_{A}^{\mathrm{epi}}\left(a_{s}, \widehat{G}\right)=\min _{\epsilon_{\|}}\left[E_{A}^{\mathrm{tot}}\left(a_{s}, \epsilon_{\|}, \widehat{G}\right)\right]-E_{A}^{\mathrm{tot}}\left(a_{A}\right) .
$$

The epitaxial strain energy $\Delta E_{A}^{\mathrm{epi}}\left(a_{s}, \widehat{G}\right)$ is related to the epitaxial softening function ${ }^{53,18} q\left(a_{s}, \widehat{G}\right)$ by the relation:

$$
q\left(a_{s}, \widehat{G}\right)=\frac{\Delta E_{A}^{\mathrm{epi}}\left(a_{s}, \widehat{G}\right)}{\Delta E_{A}^{\mathrm{bulk}}\left(a_{s}\right)}
$$

where $\Delta E_{A}^{\text {bulk }}\left(a_{s}\right)$ is the hydrostatic deformation energy of fcc $A$ to the substrate lattice constant $a_{s}$. The function Eq. (13) quantifies energy lowering due to the relaxation of $\mathbf{c}(A)$ in the second step of the deformation process considered above.

The harmonic elasticity theory without the shear strain gives ${ }^{18,56,46} q_{\text {harm }}(\widehat{G})$ which depends on the growth direction $\widehat{G}$ but not on the substrate lattice constant $a_{s}$ :

$$
q_{\mathrm{harm}}(\widehat{G})=1-\frac{B}{C_{11}+\Delta \gamma_{\mathrm{harm}}(\widehat{G})},
$$

where $B=\frac{1}{3}\left(C_{11}+2 C_{12}\right)$ is the bulk modulus, $\Delta=C_{44}-$ $\frac{1}{2}\left(C_{11}-C_{12}\right)$ is the elastic anisotropy parameter, and $\gamma_{\text {harm }}(\widehat{G})$ is a geometric function of the spherical angles formed by $\widehat{G}$ :

$$
\begin{aligned}
& \gamma_{\text {harm }}(\phi, \theta)=\sin ^{2}(2 \theta)+\sin ^{4}(\theta) \sin ^{2}(2 \phi) \\
& =\frac{4}{5} \sqrt{4 \pi}\left[K_{0}(\phi, \theta)-\frac{2}{\sqrt{21}} K_{4}(\phi, \theta)\right] .
\end{aligned}
$$

$K_{l}$ is the Kubic harmonic of angular momentum $l$. The equilibrium value of the $c_{\|} / a$ ratio of the film is given by

$c_{\|}^{\mathrm{eq}}\left(a_{s}, \widehat{G}\right)=a_{s}\left(1+\epsilon_{\|}\right)=a_{A}-\left[2-3 q_{\mathrm{harm}}(\widehat{G})\right]\left(a_{s}-a_{A}\right)$.

For the principle high-symmetry directions we have

$$
\gamma_{\text {harm }}([001])=0, \quad \gamma_{\text {harm }}([110])=1, \quad \gamma_{\text {harm }}([111])=\frac{4}{3}
$$

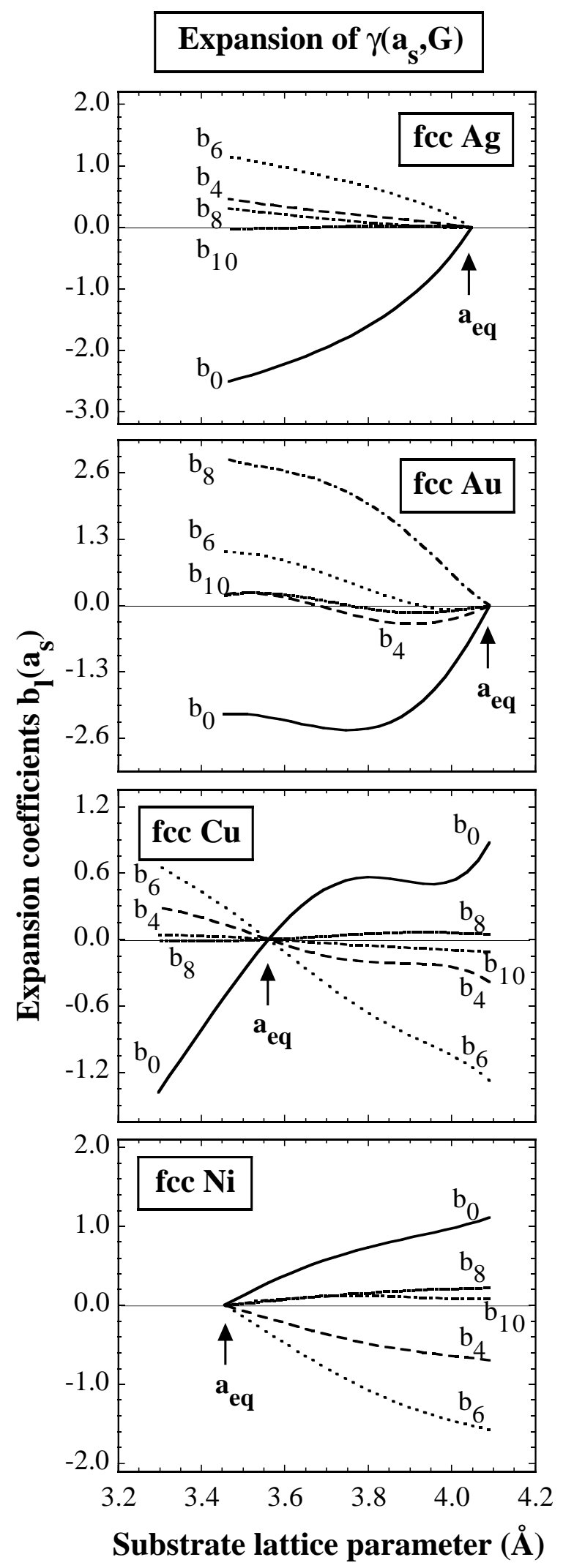

FIG. 1. Expansion coefficients $b_{l}\left(a_{s}\right)$ of Eq. (18) for Ag, $\mathrm{Au}, \mathrm{Cu}$, and $\mathrm{Ni}$. 
A parametric plot of $\gamma$ is presented in Ref. 56, which shows that the minimum of $\gamma(\widehat{G})$ is along $\langle 001\rangle$ and the maximum - along $\langle 111\rangle$. Therefore, depending on the sign of the elastic anisotropy $\Delta, q_{\text {harm }}(\widehat{G})$ is either lowest for the $\langle 001\rangle$ direction, and then $q_{\text {harm }}([111])$ is the highest, or vice versa. Other directions always have intermediate values of $q_{\mathrm{harm}}(\widehat{G})$.

If anharmonic effects are important, $q$ becomes a function of the substrate lattice parameter $a_{s}$. As we will show in Sec. III B, for deformations $2\left(a_{s}-a_{A}\right) /\left(a_{s}+a_{A}\right)$ of approximately $4 \%$, the "exact" LDA $q\left(a_{s}, \widehat{G}\right)$ exhibits appreciable dependence on the substrate lattice parameter $a_{s}$ and certain qualitative features cannot be reproduced by the harmonic functional form Eqs. (14)-(15). Furthermore, sufficiently large epitaxial strains may take the lattice from the face-centered cubic (fcc) structure into other low-energy structures [e.g., body-centered cubic (bcc) and body-centered tetragonal (bct)], causing anomalous softening of $q\left(a_{s}, \widehat{G}\right)$ for these directions. Section III B shows that this indeed happens for $\langle 001\rangle$ epitaxial strain when $a_{s}>a_{A}$. Therefore, Eqs. (14)-(15) must be generalized to account for nonlinear effects beyond the reach of the harmonic theory. This is achieved by replacing in Eq. (14) $\gamma_{\text {harm }}(\widehat{G})$ by $\gamma\left(a_{s}, \widehat{G}\right)$, where

$$
\gamma\left(a_{s}, \widehat{G}\right)=\gamma_{\mathrm{harm}}(\widehat{G})+\sum_{l=0}^{l_{\max }} b_{l}\left(a_{s}\right) K_{l}(\widehat{G})
$$

includes higher Kubic harmonics. For cubic systems $l=$ $0,4,6,8, \ldots$ The general expression for $q$ is

$$
q\left(a_{s}, \widehat{G}\right)=1-\frac{B}{C_{11}+\Delta \gamma\left(a_{s}, \widehat{G}\right)} .
$$

We have chosen this particular form for $\gamma$ since it guarantees that all expansion coefficients tend to zero in the harmonic limit:

$$
\lim _{a_{s} \rightarrow a_{A}} b_{l}\left(a_{s}\right)=0 .
$$

In summary, to calculate $\Delta E^{\mathrm{epi}}\left(a_{s}, \widehat{G}\right)$ of Eq. (3) we will use Eq. (12) to obtain it from LDA for a few substrate lattice parameters $a_{s}$ and along selected symmetry directions $\widehat{G}$. We will also need to obtain the harmonic elastic constants $C_{11}, C_{12}$ and $C_{44}$. The calculated $\Delta E^{\mathrm{epi}}\left(a_{s}, \widehat{G}\right)$ results are then fitted by the general Eqs. (13), (18) and (19).

\section{B. Anharmonic epitaxial strain of thin films of pure elements: LDA results}

We have calculated the epitaxial strain energy $\Delta E^{\text {epi }}\left(a_{s}, \widehat{G}\right)$ for $\mathrm{Cu}, \mathrm{Ni}, \mathrm{Ag}$ and $\mathrm{Au}$ along six principle directions $\langle 001\rangle,\langle 111\rangle,\langle 110\rangle,\langle 113\rangle,\langle 201\rangle$ and $\langle 221\rangle$. The local-density approximation ${ }^{58}$ (LDA), as

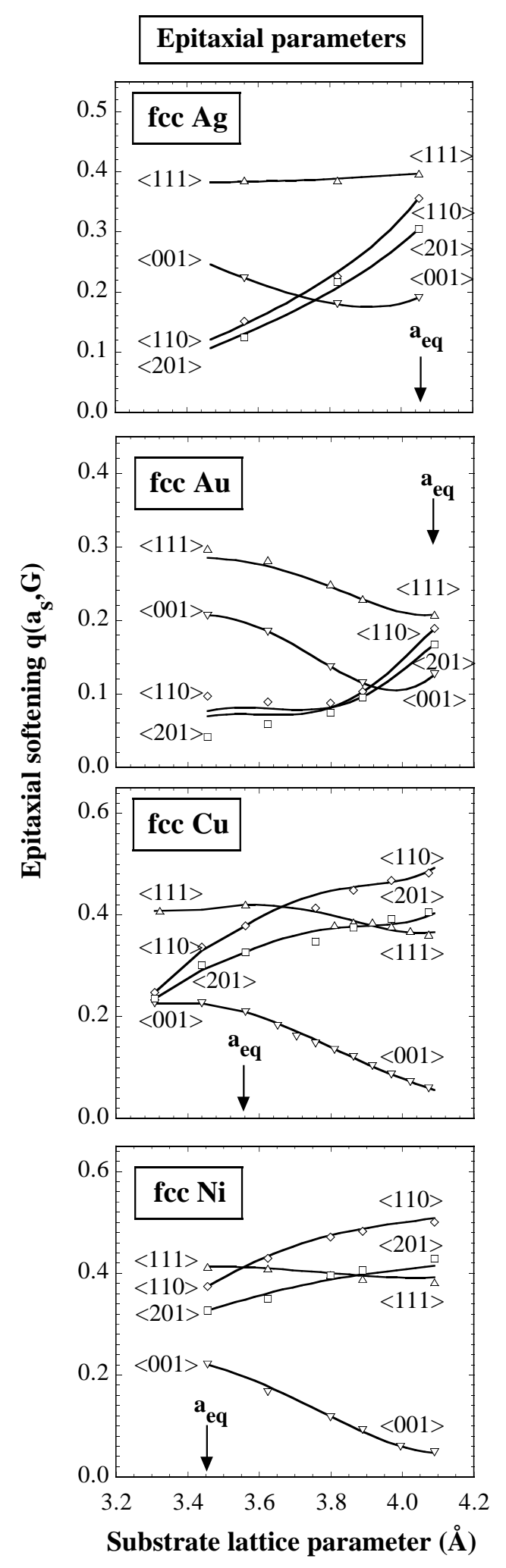

FIG. 2. The calculated epitaxial softening functions $q\left(a_{s}, \widehat{G}\right)$ for $\mathrm{Cu}, \mathrm{Ni}, \mathrm{Ag}$ and $\mathrm{Au}$. Points represent the directly calculated LDA values and lines show the fit using Eqs. (19)-(18). 


\section{Epitaxial softening $q\left(a_{S}, G\right)$}

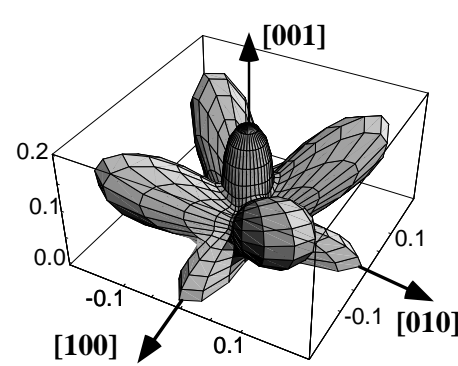

(a) $\mathrm{Au}, \mathrm{a}_{\mathrm{S}}=3.56 \AA$

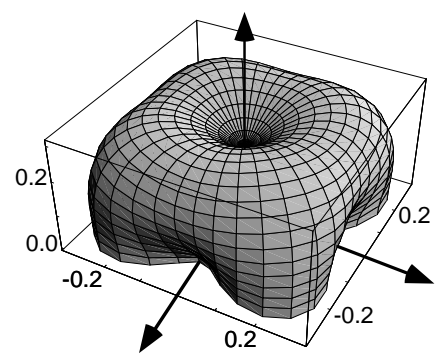

(d) $\mathrm{Cu}, \mathrm{a}_{\mathrm{S}}=3.56 \AA$ (eq)

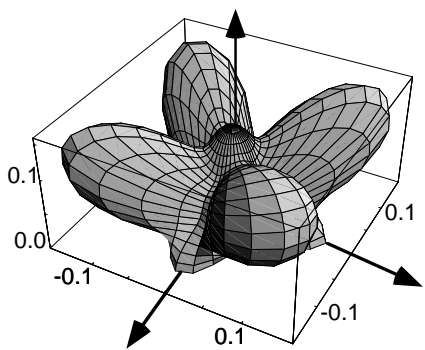

(b) $\mathrm{Au}, \mathrm{a}_{\mathrm{S}}=3.83 \AA$

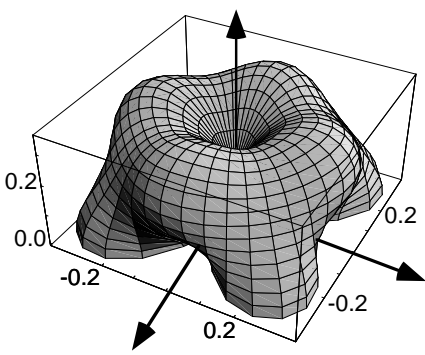

(e) $\mathrm{Cu}, \mathrm{a}_{\mathrm{S}}=3.83 \AA$

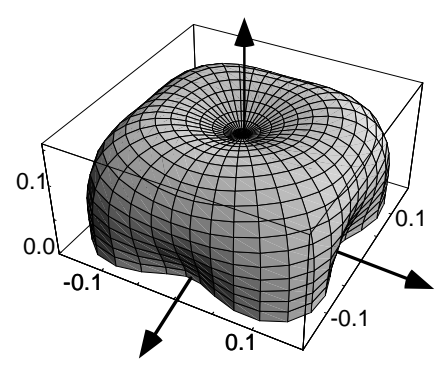

(c) $A u, a_{s}=4.09 \AA(e q)$

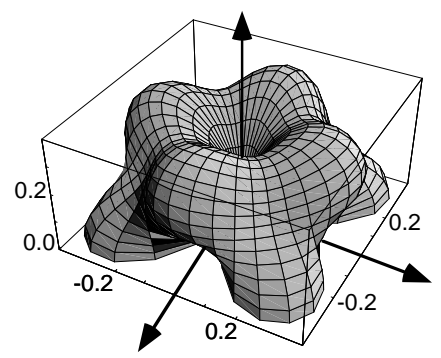

(f) $\mathrm{Cu}, \mathrm{a}_{\mathrm{S}}=4.09 \AA$

FIG. 3. Epitaxial softening function $q\left(a_{s}, \widehat{G}\right)$ for (a)-(c) $\mathrm{Cu}$ and (d)-(e) Au, at different values of the substrate lattice constant $a_{s}$.

implemented by the linearized augmented plane wave (LAPW) $\operatorname{method}^{59}$, was used to obtain the total energies in Eqs. (12) and (13). $q\left(a_{s}, \widehat{G}\right)$ was calculated from Eq. (13) and fitted with the functional form Eqs. (18)(19). The angular momentum cutoff in Eq. (18) was set to $l_{\max }=10$, leaving five independent coefficients for each value of the substrate lattice parameter $a_{s}$. This choice allows reproduction of the LDA values with a maximum error of 0.04. The calculations have been done for biaxial compression $\left(a_{s}<a_{\text {eq }}\right)$ of $\mathrm{Au}$ and $\mathrm{Ag}$, for biaxial expansion $\left(a_{s}>a_{\mathrm{eq}}\right)$ of $\mathrm{Ni}$, and for both biaxial expansion and compression of $\mathrm{Cu}$. The expansion coefficiets $b_{l}\left(a_{s}\right)$, entering Eqs. (18), are shown in Fig. 1. At the equilibrium lattice constant $a_{\text {eq }}$ (vertical arrows in Fig. 1), where the harmonic formula Eq. (15) is exact, all $b_{l}$ are exactly zero. As $a_{s}$ deviates from $a_{\mathrm{eq}}$, they change rapidly indicating the importance of anharmonic effects. In $\mathrm{Cu}$ and $\mathrm{Ni}$ for $a_{s}>a_{\mathrm{eq}}, l=6$ term is as important as $l=0$ and $l=4$ terms, contributions from $l \geq 8$ being an order of magnitude smaller. In $\mathrm{Au}$ for $a_{s}<a_{\mathrm{eq}}, b_{0}\left(a_{s}\right)$ and $b_{8}\left(a_{s}\right)$ are the dominating terms, while the behavior of $\mathrm{Ag}$ is mainly determined by $b_{0}\left(a_{s}\right)$ and $b_{6}\left(a_{s}\right)$. Thus, in spite of broad similarities between the studied elements, they exhibit some interesting differences.

Figure 2 shows the calculated LDA epitaxial softening functions $q\left(a_{s}, \widehat{G}\right)$ of Eq. (13) for $\mathrm{Cu}, \mathrm{Ni}, \mathrm{Ag}$ and $\mathrm{Au}$. There are important qualitative and quantitative differ- ences between $q_{\text {harm }}(\widehat{G})$ given by the harmonic elasticity Eq. (14), and the anharmonic $q\left(a_{s}, \widehat{G}\right)$ calculated from the LDA. First, all $q\left(a_{s}, \widehat{G}\right)$ depend on the substrate lattice constant $a_{s}$, while the harmonic $q_{\text {harm }}(\widehat{G})$ are independent of $a_{s}$. Figure 3 shows the directional dependence of $q\left(a_{s}, \widehat{G}\right)$ for $\mathrm{Cu}$ and $\mathrm{Au}$ at a few values of $a_{s}$ : equilibrium lattice parameter of $\mathrm{Cu}(3.56 \AA)$, equilibrium lattice parameter of $\mathrm{Au}(4.04 \AA)$, and halfway between them $(3.83 \AA)$. By construction, $q$ at $a_{s}=a_{\mathrm{eq}}$ is given by the harmonic form Eqs. (14)-(15), shown for fcc Au in Fig. 3(c) and fcc $\mathrm{Cu}$ in Fig. 3(d). Epitaxial deformation of $\mathrm{Au}$ with $a_{s}<a_{\mathrm{eq}}$ makes the lobes along $\langle 111\rangle$ much more pronounced than in the harmonic case. Furthermore, $q$ for Au develops additional lobes along $\langle 001\rangle$, which in the harmonic approximation is the softest direction. In contrast, $q$ of $\mathrm{Cu}$ under biaxial expansion exhibits pronounced deepening of the $\langle 001\rangle$ minima, but develops maxima along $\langle 110\rangle$.

Second, in the harmonic elasticity theory of Eq. (14) if $\langle 001\rangle$ is the softest direction (smallest $q_{\text {harm }}$ ), then $\langle 111\rangle$ must be the hardest direction, and vice versa. Figure 2 shows that this order does not hold for large deformations: the hardest direction in $\mathrm{Ni}$ and $\mathrm{Cu}$ for $a_{s} \gg a_{\text {eq }}$ is $\langle 110\rangle$, while the hardest directions in $\mathrm{Ag}$ and $\mathrm{Au}$ for $a_{s} \ll a_{\text {eq }}$ are $\langle 111\rangle$ and $\langle 001\rangle$, both $\langle 110\rangle$ and $\langle 201\rangle$ being much softer than the former. 


\section{Epitaxial (100) strain energy of $\mathrm{Cu}$}
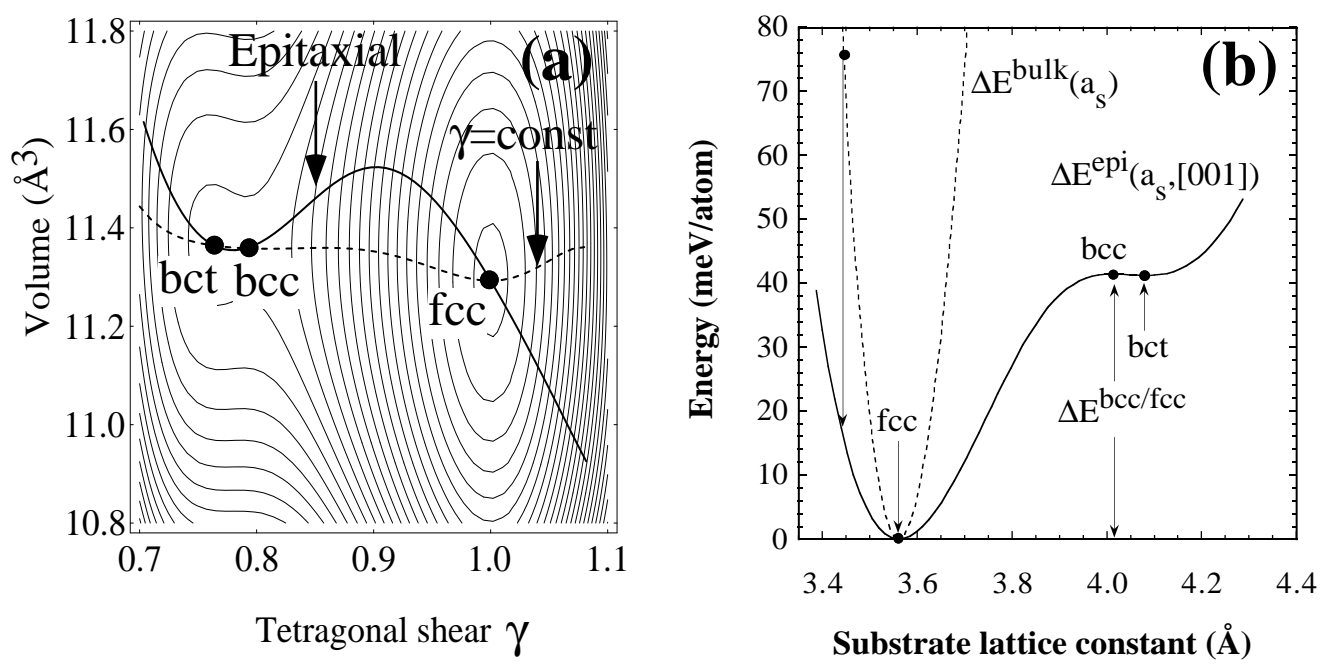

FIG. 4. Contour plot of the two-dimensional energy surface $E(\gamma, V)$ for $\mathrm{Cu}$. The continuous line shows the epitaxial path determined by Eq. (23), while the dashed line is the relation $V=V(\gamma)$ obtained by minimizing $E(\gamma, V)$ with respect to the volume $V$ at a constant $\gamma$. The right panel shows the epitaxial strain energy as a function of the substrate lattice constant in comparison with the (much larger) bulk deformation energy $\Delta E^{\mathrm{bulk}}\left(a_{s}\right)$.

Third, Fig. 2 shows that $q\left(a_{s}, \widehat{G}\right)$ of different directions cross for substrate/film lattice mismatch $2\left|a_{s}-a_{\mathrm{eq}}\right| / \mid a_{s}+$ $a_{\text {eq }} \mid<4 \%$. For example, while $\langle 001\rangle$ is the softest direction near $a_{\text {eq }}$ and stays such upon biaxial expansion $(\mathrm{Cu}$, $\mathrm{Ni}$ ), it is one of the hardest in biaxially compressed metals $(\mathrm{Ag}, \mathrm{Au}, \mathrm{Cu})$ where $\langle 201\rangle$ is the softest direction. Similarly, $\langle 111\rangle$ is the hardest direction near the equilibrium and for $a_{s} \ll a_{\text {eq }}$, but it becomes softer than $\langle 110\rangle$ and $\langle 201\rangle$ in biaxially expanded $\mathrm{Cu}$ and $\mathrm{Ni}$. Thus, there is a qualitative breakdown of the harmonic theory for strains of $4 \%$, and presumably quantitative errors for even smaller strains.

We also note similarities in the elastic behavior of these materials. Under expansion, both $\mathrm{Cu}$ and $\mathrm{Ni}$ exhibit strong softening of $q\left(a_{s},[001]\right)$ and somewhat weaker softening of $q\left(a_{s},[111]\right)$, while $q\left(a_{s},[110]\right)$ becomes the elastically hardest direction. This order is reversed under biaxial compression of $\mathrm{Ag}, \mathrm{Au}$ and $\mathrm{Cu}$ : $q$ 's for $\langle 001\rangle$ and $\langle 111\rangle$ harden, but the $\langle 110\rangle$ and $\langle 201\rangle$ directions soften.

\section{Discussion of anomalous softening of $q\left(a_{s}, \widehat{G}\right)$ in terms of fcc/bcc energy differences}

The anomalous softening of $q\left(a_{s},[001]\right)$ in $\mathrm{Ni}$ and $\mathrm{Cu}$ for $a_{s}>a_{\text {eq }}$ reflects a small fcc/bcc energy difference for these materials. This can be seen by considering three energy surfaces that deform fcc into bcc:

(i) $E(\gamma, V)$ : The most general surface is the total energy as a function of the tetragonal shear $\gamma$ and volume
$V$, shown as contour in Fig. 4(a) for $\mathrm{Cu}$. The tetragonal shear along $\langle 001\rangle$ is defined by:

$$
\varepsilon_{i j}=\left(\begin{array}{ccc}
\gamma & 0 & 0 \\
0 & \gamma^{-\frac{1}{2}} & 0 \\
0 & 0 & \gamma^{-\frac{1}{2}}
\end{array}\right),
$$

where $c / a=\sqrt{2} \gamma^{\frac{3}{2}} . E(\gamma, V)$ has (at least) three extremal points, denoted in Fig. 4(a) as solid dots: one corresponding to the fcc state, one to the bcc state and one to the bct state. These states obey the extremal conditions of vanishing derivatives:

$$
\frac{\partial}{\partial \gamma} E(\gamma, V)=\frac{\partial}{\partial V} E(\gamma, V)=0
$$

Figure 4(a) shows that for $\mathrm{Cu}$ fcc and bct are locally stable minima with respect to $\gamma$ and $V$, while bcc is a saddle point (maximum with respect to $\gamma$ and minimum with respect to $V) \cdot{ }^{64,65}$

(ii) Bain path $E(\gamma)$ : A more specific function $E(\gamma) \equiv$ $\left.E(\gamma, V)\right|_{V=\text { const }}$ is defined by the tetragonal Bain path, ${ }^{63}$ connecting fcc and bcc structures. The Bain path is obtained by changing the $c / a$ ratio while keeping $V=c a^{2}$ constant. When $c / a=1$ the lattice type is bcc and when $c / a=\sqrt{2}$ it is fcc. The energy as a function of $\gamma$ must have extremal points at both $\gamma$ values corresponding to the cubic symmetry fcc $\left(\gamma_{\mathrm{fcc}}=1\right)$ and bcc $\left(\gamma_{\mathrm{bcc}}=2^{-\frac{1}{3}}\right)$ states, as well as at least another bct point $\gamma_{\text {bct }}$ with a zero derivative $E^{\prime}(\gamma)=0 .{ }^{66,67}$ Usually, ${ }^{64-66,68-70}$ for fcc stable elements the bcc lattice is unstable [i.e., $E(\gamma)$ 
has a local maximum at $\gamma_{\mathrm{bcc}}$ ] and the bct state (a local minimum) occurs for $\gamma_{\mathrm{bct}}<\gamma_{\mathrm{bcc}}$.

(iii) Epitaxial Bain path $E\left[c_{\mathrm{eq}}\left(a_{s}\right)\right]$ : This deformation path is obtained by scanning $c$ while $a_{s}$ is kept fixed, which corresponds to epitaxial growth on a (001) substrate with lattice parameter $a_{s} . c$ is determined from the total energy minimization at a fixed $a_{s}$ :

$$
\frac{d}{d c} E^{\mathrm{tot}}(\gamma, V)=\left(\frac{2}{3} \gamma^{-\frac{1}{3}} \frac{\partial}{\partial \gamma}+a_{s}^{2} \frac{\partial}{\partial V}\right) E(\gamma, V)=0 .
$$

Eq.(23) defines the epitaxial path $V(\gamma)$, shown as a continuous line in Fig. 4(a). Since $c / a_{s}=\sqrt{2} \gamma^{\frac{3}{2}}$ and $V=c a_{s}^{2} / 4$, this path implicitly relates the out-of-plane dimension $c$ to the substrate lattice constant $a_{s}$, much like Eq. (16) does in the harmonic case. As noted in Ref. 65, the epitaxial path crosses all extremal points of $E(\gamma, V)$ because Eq. (23) is satisfied where conditions Eq. (22) hold. Therefore, if we parametrize the epitaxial strain energy along this path as a function of $a_{s}$, it has a global minimum corresponding to fcc, a locally stable minimum corresponding to bct and a maximum at the bcc state, see Fig. 4(b). We see that as $a_{s}$ increases from the equilibrium fcc value, $\mathrm{Cu}$ sequentially passes through the bcc and bct states where the strain energy $\Delta E^{\mathrm{epi}}\left(a_{s},[001]\right)$ is equal to the fcc/bcc and fcc/bct structural energy differences. When these energy differences are much smaller than the characteristic values of the bulk deformation energies $\Delta E^{\mathrm{bulk}}\left(a_{s}\right)$ [see Fig. 4(b)], then $q\left(a_{s},[001]\right)$ is anomalously soft [since $q\left(a_{s},[001]\right)=\Delta E^{\mathrm{bcc} / \mathrm{fcc}} / \Delta E_{\mathrm{fcc}}^{\mathrm{bulk}}\left(a_{s}\right)$ for $\left.a_{s}=\left(2 V_{\mathrm{bcc}}\right)^{\frac{1}{3}}\right]$.

In summary, the softness of $q\left(a_{s},[001]\right)$ for $a_{s}>a_{\mathrm{eq}}$ is a reflection of the geometric properties of the $\langle 001\rangle$ epitaxial deformation path (connection between cubic symmetry fcc and bcc structures), and a small fcc/bcc energy difference, $\Delta E^{\mathrm{fcc} / \mathrm{bcc}} \ll \Delta E^{\mathrm{bulk}}\left(a_{s}\right)$. It is important that the fcc and bcc points correspond to lattices with cubic symmetry, since it ensures that the energy surface has extremal points there. In zincblende $\mathrm{GaP}$ and $\mathrm{InP},{ }^{71}$ epitaxial $\langle 001\rangle$ path has only one point of cubic symmetry $(c / a=\sqrt{2}$, corresponding to undistorted fcc), and therefore the energy surface $E(\gamma, V)$ is not required to possess additional extremal points. As a consequence, $\Delta E^{\mathrm{epi}}\left(a_{s},[001]\right)$ is a monotonously increasing function of $a_{s}$, and $q\left(a_{s},[001]\right)$ does not soften with increasing $a_{s}$.

The described mechanism also accounts for the softening of $q\left(a_{s},[111]\right)$ for $a_{s}>a_{\mathrm{eq}}$ in $\mathrm{Cu}$ and $\mathrm{Ni}$ under biaxial $\langle 111\rangle$ expansion, since this deformation takes fcc $(c / a=\sqrt{6})$ into bcc $(c / a=\sqrt{6} / 4)$, albeit at a much larger strain. However, we have not found any simple structure corresponding to the compressive $\langle 201\rangle$ strain which could explain the softening of $q\left(a_{s}<a_{\mathrm{eq}}\right.$, [201]) in $\mathrm{Ag}, \mathrm{Au}$ and $\mathrm{Cu}$. The latter seems to be caused by relatively loose packing of atoms within the (201) planes, imposing small energy penalty on decreasing the interatomic distances. Indeed, the nearest-neighbor distance in (201) plane is $a_{s}$, compared to $a_{s} / \sqrt{2}$ in (111) or (001) planes with high values of $q\left(a_{s}, \widehat{G}\right)$ for $a_{s}<a_{\text {eq }}$.

\section{STABILITY OF SUPERLATTICES AND ALLOYS}

\section{A. Constituent strain of superlattices}

The bulk formation enthalpy of superlattices [Eq. (8)] is expressed as a sum of the interfacial energy $I(n, \widehat{G})$ and constituent strain energy $\Delta E_{\mathrm{CS}}^{\mathrm{eq}}(x, \widehat{G})$. As given by Eq. (6), the latter is a weighted average of the epitaxial strain energies of coherently strained constituents, minimized with respect to the common in-plane lattice vectors $\mathbf{a}_{1}$ and $\mathbf{a}_{2}$. For the high symmetry directions $\langle 001\rangle$ and $\langle 111\rangle$, these vectors are related by symmetry operations of the superlattice, so that $\mathbf{a}_{1}$ and $\mathbf{a}_{2}$ are proportional to the ideal fcc unit vectors $\mathbf{a}_{1}^{0}$ and $\mathbf{a}_{2}^{0}$ via Eq. (2). Then $\Delta E_{\mathrm{CS}}^{\mathrm{eq}}(x, \widehat{G})$ can be calculated by minimizing the following expression with respect to the superlattice parameter $a_{\mathrm{SL}}$ :

$$
\begin{aligned}
\Delta E_{\mathrm{CS}}^{\mathrm{eq}}(x, \widehat{G})=\min _{a_{\mathrm{SL}}}[ & (1-x) \Delta E_{A}^{\mathrm{epi}}\left(a_{\mathrm{SL}}, \widehat{G}\right) \\
& \left.+x \Delta E_{B}^{\mathrm{epi}}\left(a_{\mathrm{SL}}, \widehat{G}\right)\right] .
\end{aligned}
$$

For lower symmetry directions $\widehat{G}$, the in-plane unit vectors $\mathbf{a}_{1}$ and $\mathbf{a}_{2}$ may relax differently, and the angle $\cos \gamma=\mathbf{a}_{1} \cdot \mathbf{a}_{2} /\left|\mathbf{a}_{1}\right|\left|\mathbf{a}_{2}\right|$ is also free to vary. For instance, in $\langle 110\rangle$ superlattices, the vectors $\mathbf{a}_{1}$ and $\mathbf{a}_{2}$ are not related by symmetry, and therefore may scale differently, i.e., in ideal fcc $\left|\mathbf{a}_{1}^{0}\right| /\left|\mathbf{a}_{2}^{0}\right|=\sqrt{2}$ but in the superlattice generally $\left|\mathbf{a}_{1}\right| /\left|\mathbf{a}_{2}\right| \neq \sqrt{2}$. Equation (24) is much simpler than the general Eq. (6) requiring minimization with respect to three degrees of freedom: lengths $\left|\mathbf{a}_{1}\right|,\left|\mathbf{a}_{2}\right|$, and the angle $\alpha=\left(\widehat{\mathbf{a}_{1}, \mathbf{a}_{2}}\right)$. In the present work we adopt Eq. (24) even for low symmetry directions, using the calculated $\Delta E_{A}^{\mathrm{epi}}\left(a_{s}, \widehat{G}\right)$ from Sec. III B.

$\Delta E_{\mathrm{CS}}^{\mathrm{eq}}$ : Figure 5 shows the equilibrium constituent strain energies $\Delta E_{\mathrm{CS}}^{\mathrm{eq}}(x, \widehat{G})$ for the size-mismatched $\mathrm{Cu}-$ $\mathrm{Ag}, \mathrm{Ni}-\mathrm{Au}$ and $\mathrm{Cu}-\mathrm{Au}$ systems. They are determined from Eq. (24), using only the epitaxial strain energies $\Delta E_{A, B}^{\mathrm{epi}}$ of the constituents. There are obvious similarities in $\Delta E_{\mathrm{CS}}^{\mathrm{eq}}(x, \widehat{G})$ for the three noble metal systems. $\langle 201\rangle$ superlattices have the lowest constituent strain energy below $x \approx 0.2$, after that $\langle 001\rangle$ becomes the softest direction. $\langle 111\rangle$ is the hardest direction over a wide composition range, except close to $x=1$ where $\langle 110\rangle$ is slightly harder.

This behavior can be explained by the properties of the epitaxial softening function $q\left(a_{s}, \widehat{G}\right)$, discussed in Sec. III B. For example, consider $\mathrm{Cu}-\mathrm{Au}$ from Fig. 5 . Upon biaxial compression of $\mathrm{Au}$ (corresponding to $x<$ $0.5), q\left(a_{s},[111]\right)$ increases rapidly (see Fig. 2), increasing the elastic strain energy and making this an elastically hard direction. In contrast, $q\left(a_{s},[201]\right)$ for Au decreases with biaxial compression, and at $x<0.2$ there is small energetic penalty for deforming $\mathrm{Cu}$ and $\mathrm{Au}$ to a common in-plane lattice constant. Increase of $q\left(a_{s},[110]\right)$ for $\mathrm{Cu}$ 


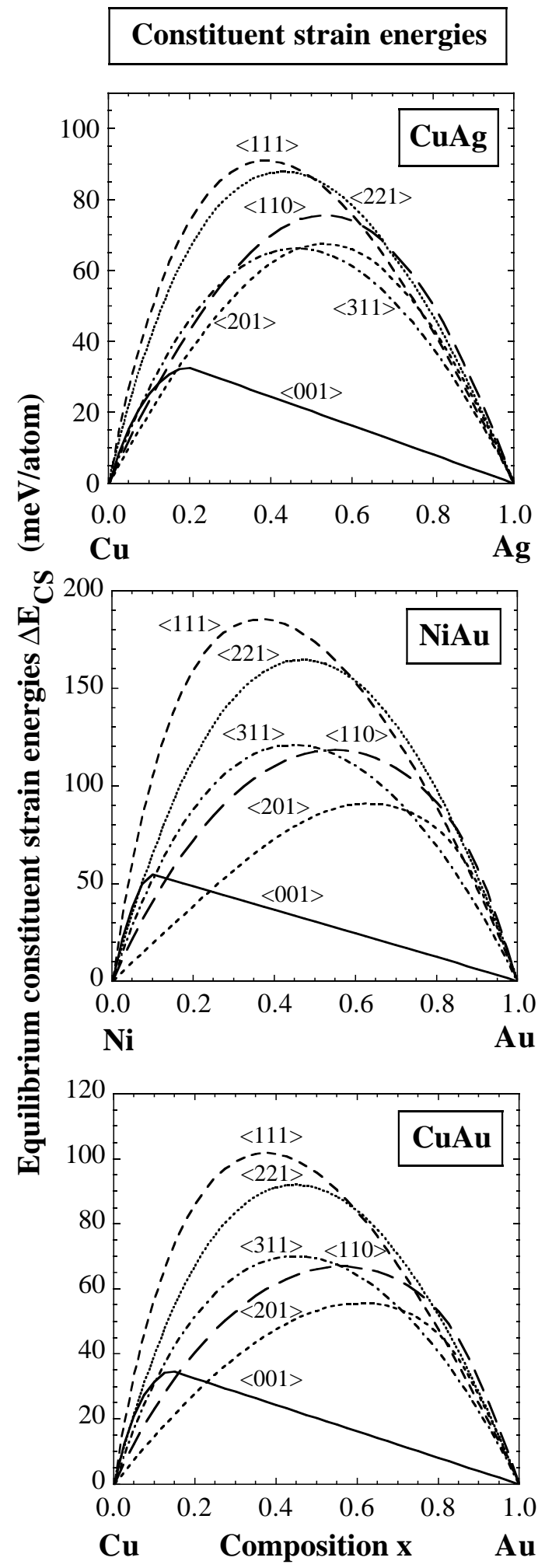

FIG. 5. Equilibrium constituent strain energies for $\mathrm{Cu}-\mathrm{Au}$, $\mathrm{Ni}-\mathrm{Au}$ and $\mathrm{Cu}-\mathrm{Ag}$. $\mathrm{Ag}-\mathrm{Au}$ system is size matched and $\Delta E_{\mathrm{CS}}^{\mathrm{eq}}=0$.

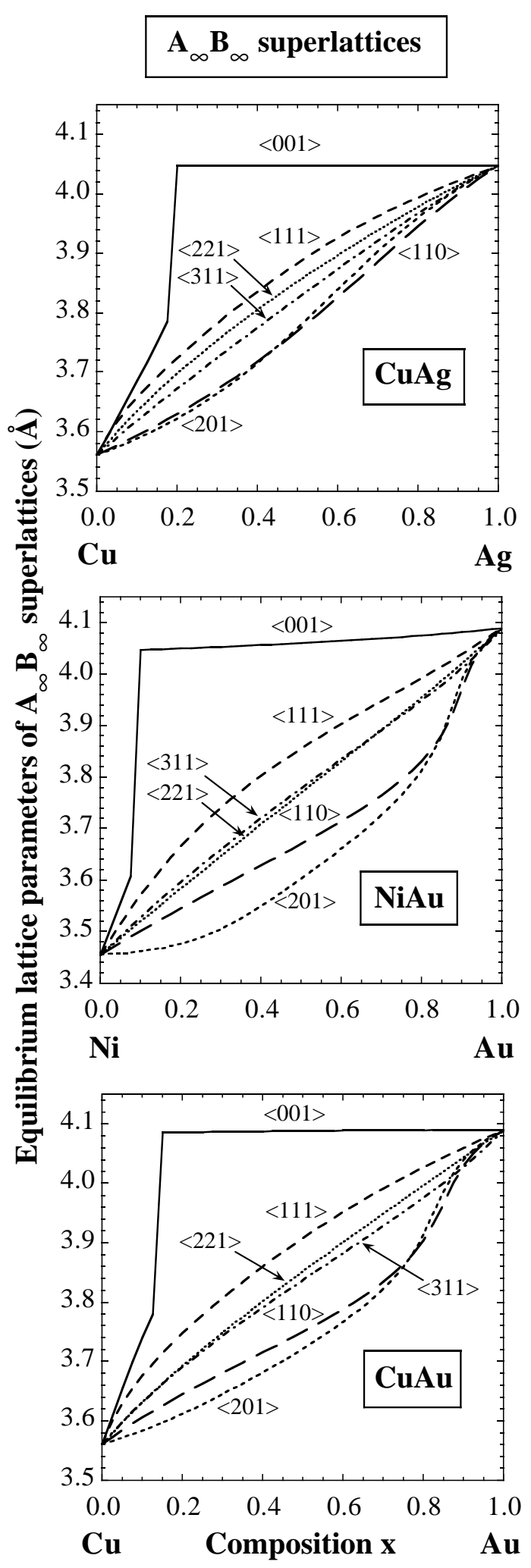

FIG. 6. Equilibrium lattice parameter of infinite $\mathrm{Cu}-\mathrm{Au}$, $\mathrm{Cu}-\mathrm{Ag}$ and $\mathrm{Ni}-\mathrm{Au}$ superlattices vs composition. 
with $a_{s}$ eventually causes this to be the hardest direction in $\mathrm{Au}$-rich $\mathrm{Cu}-\mathrm{Au}$ superlattices.

$a_{\mathrm{SL}}(x)$ : Figure 6 shows the equilibrium in-plane lattice constant $a_{\mathrm{SL}}(x, \widehat{G})$ that minimizes the constituent strain. These are also the equilibrium lattice parameters for infinite period superlattices. The lattice parameters $a_{\mathrm{SL}}(x, \widehat{G})$ show large deviations from Vegard's law, with the behavior of $a_{\mathrm{SL}}(x,[001])$ being particularly anomalous. The very unusual composition dependence of the superlattice parameter for $\langle 001\rangle$ deserves a closer scrutiny: At $x \approx 0.2$ the superlattice parameter changes discontinuously to the lattice parameter of the larger constituent. The constituent strain energy abruptly changes slope and settles down to a strictly linear composition dependence. Furthermore, $\Delta E_{\mathrm{CS}}^{\mathrm{eq}}(x,[001])$ is very small in comparison with $\Delta E_{\mathrm{CS}}^{\mathrm{eq}}$ for other directions. These anomalies are direct consequences of the soft $q\left(a_{s},[001]\right)$ for biaxially expanded $\mathrm{Cu}$ and $\mathrm{Ni}$, which in turn is a consequence of the small fcc/bcc and fcc/bct energy differences for these metals (Sec. IIIB). Indeed, for a sufficiently Au-rich system $\Delta E_{\mathrm{Cu}}^{\mathrm{epi}}$ is smaller than $\Delta E_{\mathrm{Au}}^{\mathrm{epi}}$ favoring a superlattice constant close to the equilibrium lattice parameter of $\mathrm{Au}$. This large lattice parameter happens to fall on the flat region of the strain energy curve around the bcc and bct states of biaxially expanded $\mathrm{Cu}$ (see Fig. 4), where a local bct minimum exists in the function on the right-hand side of Eq. (24), shifting downward in energy with increasing $x$. At some critical value of the composition, the minimum around $a_{\mathrm{Au}}$ becomes deeper than the minimum close to $a_{\mathrm{Cu}}$, which causes a discontinuous jump in $a_{\mathrm{SL}}$. Loosely speaking, $\mathrm{Cu}$ deforms all the way into the bct structure and $\mathrm{Au}$ does not deform at all. That also explains the linear decrease of $\Delta E_{\mathrm{CS}}(x,[001])$ after the discontinuity, since $\Delta E_{\mathrm{Au}}^{\mathrm{epi}}=0$ and $\Delta E_{\mathrm{Cu}}^{\mathrm{epi}}=$ const in Eq. (24).

In conclusion, we summarize the prerequisites for low elastic strain energy of infinite superlattices:

(i) One of the components should exhibit a particularly soft elastic direction under biaxial strain, e.g., $\langle 001\rangle$ in $\mathrm{Cu}$ upon epitaxial expansion and $\langle 201\rangle$ in $\mathrm{Au}$ upon biaxial compression.

(ii) The lattice mismatch between the constituents should be large enough to explore the regions of anomalous softness.

We stress that the unusual behavior shown in Figs. 6 and 5 (crossing of different directions, discontinuities, different skewnesses of $\Delta E_{\mathrm{CS}}^{\mathrm{eq}}(x, \widehat{G})$ curves $)$ are caused by the anharmonic $q\left(a_{s}, \widehat{G}\right)$, and cannot be obtained within the harmonic theory with lattice parameter independent $q_{\text {harm }}(\widehat{G}) .{ }^{56}$

\section{B. Describing chemical interactions via the mixed-space cluster expansion}

The energy of a bulk alloy $\Delta H_{\mathrm{mix}}^{\mathrm{bulk}}(x)$ of Eq. (5), and of an epitaxial alloy $\Delta H_{\mathrm{mix}}^{\mathrm{epi}}(x)$ of Eq. (11) cannot be computed directly from LDA since configurationally random structures are involved. The approximate approach is either large supercells or a first-principles mixed-space cluster expansion. ${ }^{56,57}$ In the latter approach, a spin variable $S_{i}$ is assigned to each lattice site $\mathbf{R}_{i}$ which takes a value +1 if the site is occupied by an atom of type $A$, or -1 if the site is occupied by an atom of type $B$. The formation enthalpy of an arbitrary structure $\sigma$ is expressed in the following form:

$$
\begin{array}{r}
\Delta H_{\mathrm{CE}}(\sigma)=\sum_{\mathbf{k}} J_{\text {pair }}(\mathbf{k})|S(\mathbf{k}, \sigma)|^{2} \\
+\sum_{f}^{\mathrm{MB}} D_{f} J_{f} \bar{\Pi}_{f}(\sigma)+\Delta E_{\mathrm{CS}}(\sigma) .
\end{array}
$$

where $J(\mathbf{k})$ is the Fourier transform of real-space pair interactions and $S(\mathbf{k}, \sigma)$ is the structure factor,

$$
\begin{array}{r}
J_{\text {pair }}(\mathbf{k})=\sum_{j} J_{\text {pair }}\left(\mathbf{R}_{i}-\mathbf{R}_{j}\right) e^{-i \mathbf{k} \mathbf{R}_{j}} \\
S(\mathbf{k}, \sigma)=\sum_{j} S_{j} e^{-i \mathbf{k} \mathbf{R}_{j}}
\end{array}
$$

The second sum in Eq. (25) runs over symmetry inequivalent clusters constituted by three or more lattice sites. $D_{f}$ is the number of equivalent clusters per lattice site, and $\bar{\Pi}_{f}(\sigma)$ are structure-dependent geometrical coefficients (simple lattice averages of the cluster spin products). The last term in Eq. (25) is the constituent strain energy $\Delta E_{C S}(\sigma)$ of the structure $\sigma$. It is designed to reproduce the elastic strain energy of coherent longperiod superlattices ${ }^{56}$ which are calculated directly from the LDA (see Sec. IV A):

$$
\begin{array}{r}
\Delta E_{\mathrm{CS}}(\sigma)=\sum_{\mathbf{k}} J_{\mathrm{CS}}(x, \widehat{k})|S(\mathbf{k}, \sigma)|^{2}, \\
J_{\mathrm{CS}}(x, \widehat{k})=\frac{\Delta E_{\mathrm{CS}}^{\mathrm{eq}}(x, \widehat{k})}{4 x(1-x)} .
\end{array}
$$

The equilibrium constituent strain energies $\Delta E_{\mathrm{CS}}^{\mathrm{eq}}(x, \widehat{k})$ have been deduced from the directly calculated $\Delta E^{\text {epi }}\left(a_{\mathrm{SL}}, \widehat{G}\right)$ minimizing Eq. (24) with respect to the common in-plane lattice constant $a_{\mathrm{SL}}$. They are fitted by series of Kubic harmonics with composition dependent coefficients $c_{l}(x)$ :

$$
\Delta E_{\mathrm{CS}}(x, \widehat{G})=\sum_{l=0}^{l_{\max }} c_{l}(x) K_{l}(\widehat{G}),
$$

which are used to evaluate $\Delta E_{\mathrm{CS}}(x, \widehat{G})$ for any direction $\widehat{G}$. Structure factors $S(\mathbf{k}, \sigma)$ in the long-period superlattice limit are nonzero only for $\mathbf{k} \rightarrow 0$, where $J_{\mathrm{CS}}(x, \widehat{k})$ 
is a nonanalytic function of $\mathbf{k}$, reflecting the directional dependence of the constituent strain energy.

The effective cluster interactions $J_{f}$ and $J_{\text {pair }}(\mathbf{k})$ are determined by fitting Eq. (25) to a large number (30 to 40) fully relaxed first-principles LDA formation enthalpies of simple ordered structures. Most of these ordered structures are short-period superlattices along $\langle 001\rangle,\langle 111\rangle,\langle 110\rangle,\langle 201\rangle$ and $\langle 113\rangle .{ }^{60}$ The calculations of $T=0$ total energies employ the full-potential linearized augmented plane wave method ${ }^{62}$ (FLAPW) and local density approximation (LDA) for the electronic exchange and correlation. The total energy is minimized with respect to all structural degress of freedom, i.e. both the atomic positions and cell-external coordinates are fully relaxed. Complete discussion of the LDA calculations and cluster expansions for $\mathrm{Ag}-\mathrm{Au}, \mathrm{Cu}-\mathrm{Ag}, \mathrm{Cu}-\mathrm{Au}$ and $\mathrm{Ni}-\mathrm{Au}$ can found in Ref. 60.

\section{Stability of finite period metal superlattices}

Having obtained all ingredients of $\Delta H_{\mathrm{CE}}(\sigma)$ [Eq. (25)] from LDA calculations on small unit cell structures, we can use this equation to predict the energy of any configuration $\sigma$, in particular superlattices. Figure 7 shows the bulk formation energies of $(A)_{n} /(B)_{n}$ superlattices for the studied noble metal systems. The interfacial energies $I(n, \widehat{G})$, extracted from $\Delta H_{S L}(n, \widehat{G})$ according to Eq. (8), are shown in Fig. 8. Several interesting observations can be made from these figures:

(i) $I(n, \widehat{G})$ are approximately constant after $n>5$.

(ii) For ordering systems $(\mathrm{Cu}-\mathrm{Au}$ and $\mathrm{Ag}-\mathrm{Au})$, the interfacial energies are negative (see Fig. 8). Negative interfacial energies are the cause for the upward slope of $\Delta H_{\mathrm{SL}}(n, \widehat{G})$ curves in Fig. 7. This indicates a chemical preference for having unlike atoms at the interface and a tendency to form ordered structures at $T=0$. For instance, $L 1_{0}$, the observed ground state of $\mathrm{CuAu}$, is a monolayer $(\mathrm{Cu}) /(\mathrm{Au})$ superlattice along $\langle 001\rangle$. The order of $\Delta H_{\mathrm{SL}}(n, \widehat{G})$ is lowest $\langle 001\rangle$ and highest $\langle 111\rangle$ for $\mathrm{Cu}-\mathrm{Au}$, and lowest $\langle 110\rangle$ and highest $\langle 111\rangle$ for $\mathrm{Ag}-\mathrm{Au}$ superlattices.

(iii) For the phase separating $\mathrm{Cu}-\mathrm{Ag}$, all interfacial energies are positive. $\Delta H_{\mathrm{SL}}(n, \widehat{G})$ decreases with $n$ for all directions and reflect the tendency to coherent phase separation over ordered superlattice formation. Interfaces between $\mathrm{Cu}$ and $\mathrm{Ag}$ are energetically very costly. The order of $\Delta H_{\mathrm{SL}}(n, \widehat{G})$ is again lowest $\langle 001\rangle$ and highest $\langle 111\rangle$.

(iv) $\mathrm{Ni}-\mathrm{Au}$ has the most interesting behavior of $\Delta H_{\mathrm{SL}}(n, \widehat{G})$ and $I(n, \widehat{G})$. It exibits phase-separating type $\Delta H_{\mathrm{SL}}(n,[001])$ (decreasing with $n$ ), ordering type $\Delta H_{\mathrm{SL}}(n,[110])$ (increasing with $n$ ), and a nearly constant $\Delta H_{\mathrm{SL}}(n,[111])$. Does this mean that interfaces in some directions are energetically favorable, while in other directions they are energetically costly? The answer is: No. In $\mathrm{Ni}-\mathrm{Au}$, just like in $\mathrm{Cu}-\mathrm{Ag}$, all isolated

\section{Superlattice energies}
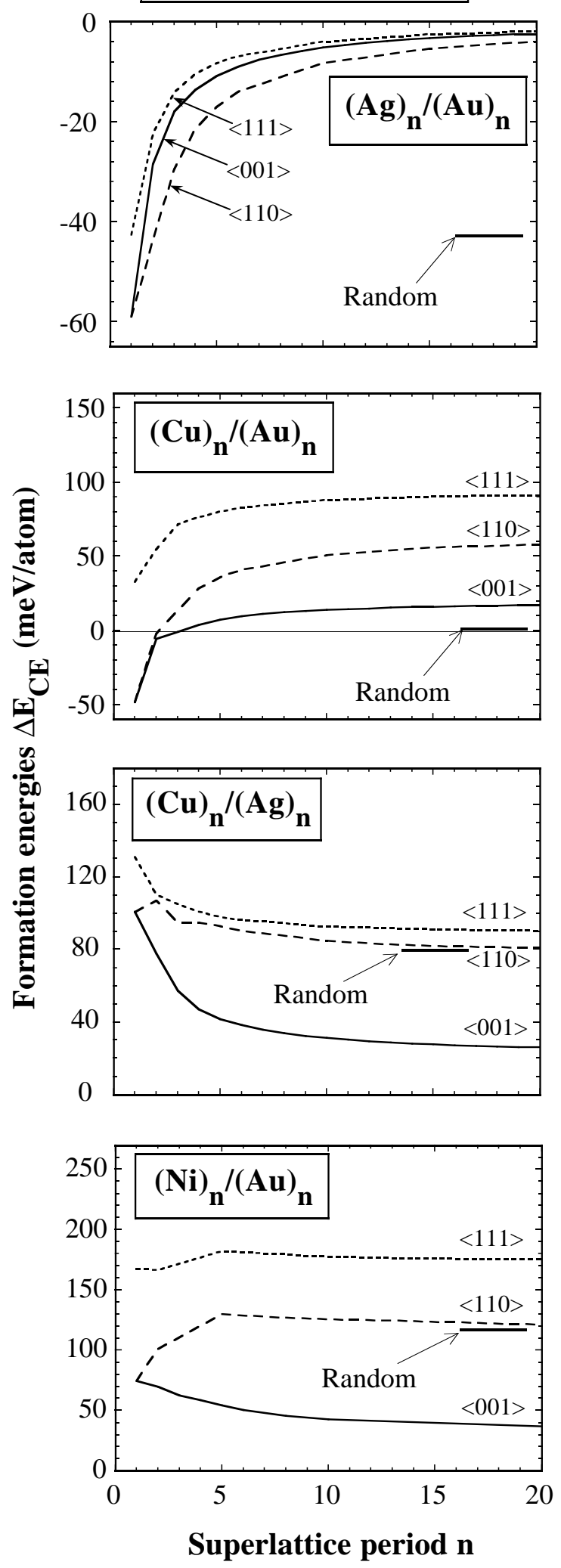

FIG. 7. Superlattice energies for $\mathrm{Cu}-\mathrm{Au}, \mathrm{Cu}-\mathrm{Ag}, \mathrm{Ni}-\mathrm{Au}$ and Ag-Au. 

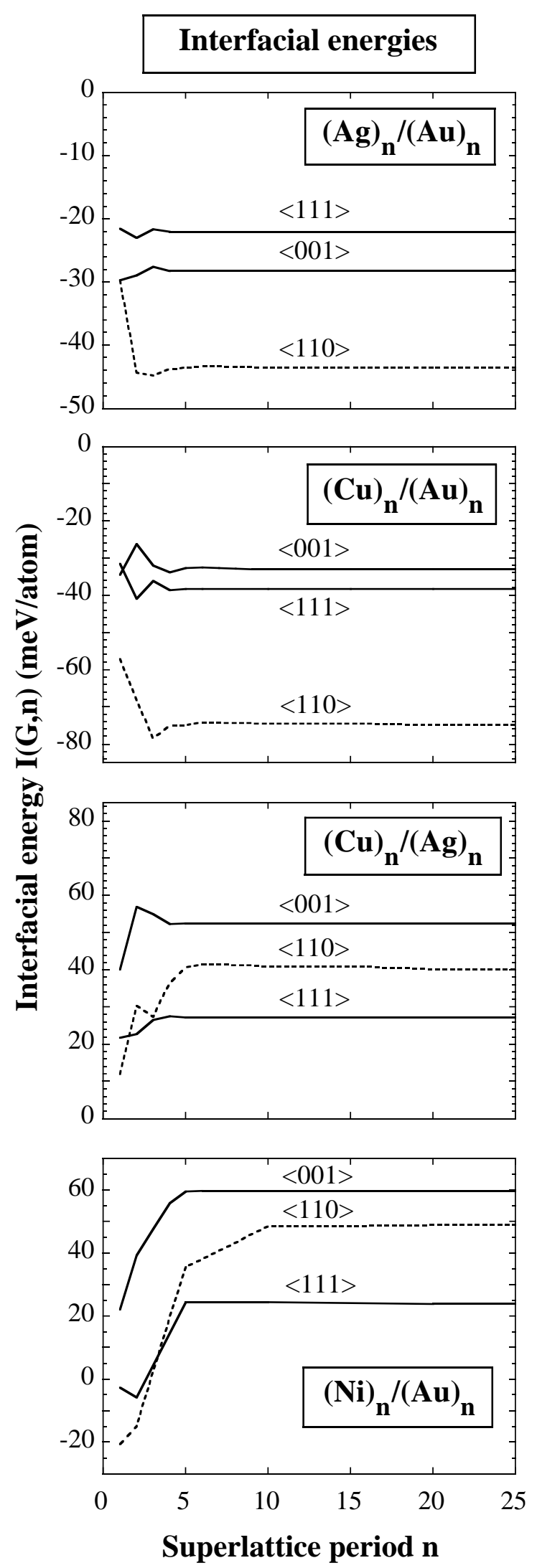

FIG. 8. Interfacial energies of $\mathrm{Cu}-\mathrm{Au}, \mathrm{Cu}-\mathrm{Ag}, \mathrm{Ni}-\mathrm{Au}$ and $\mathrm{Ag}-\mathrm{Au}$. interfaces have positive formation energies. However, the interaction between the interfaces along $\langle 110\rangle$ is strongly attractive in $N i$-Au, and leads to a net chemical energy gain for some short-period superlattices. Indeed, Fig. 8 shows that all interfacial energies of $\mathrm{Ni}-\mathrm{Au}$ are positive in the limit $n \rightarrow \infty$ (when there is no interaction between the interfaces), but decrease for short periods and are negative for $\langle 110\rangle n \leq 3$ superlattices. As we show in Ref. 61 the competition between the constituent strain energy, interfacial energy $I(n \rightarrow \infty, \widehat{G})$ and ordering-type interaction between the interfaces leads to unusual shortrange order in $\mathrm{Ni}-\mathrm{Au}$ solid solutions.

(v) It is interesting that in the phase separating $\mathrm{Ni}-\mathrm{Au}$ and $\mathrm{Cu}-\mathrm{Ag}$ the lowest interfacial energy $I(n \rightarrow \infty, \widehat{G})$ occurs for the close-packed $\{111\}$ interfaces, and the highest for $\{001\}$ interfaces. This situation is completely different in the ordering systems $\mathrm{Cu}-\mathrm{Au}$ and $\mathrm{Ag}-\mathrm{Au}$, which have $\{110\}$ as the lowest and either $\{111\}$ or $\{001\}$ as the highest $I(n \rightarrow \infty, \widehat{G})$.

(vi) Figure 7 shows the enrgies of the random alloys at the equiatomic composition. We see that in $\mathrm{Cu}-\mathrm{Au}$ and Ag-Au all long-period superlattices are unstable with respect to the formation of a random alloy. In $\mathrm{Ni}-\mathrm{Au}$ the random alloy is less favorable than coherent phase separation in the $\langle 001\rangle$ direction, but slightly more favorable than infinite coherent superlattices along $\langle 110\rangle$ and $\langle 111\rangle$. However, short-period $\langle 110\rangle$ superlattices are lower in energy than the random alloy. All $\langle 111\rangle$ superlattices of $\mathrm{NiAu}$ have higher formation enthalpies than the random alloy. In $\mathrm{Cu}-\mathrm{Ag}$ only the long-period $\langle 001\rangle$ superlattices have lower bulk formation enthalpies than the random alloy. The epitaxial growth of $\mathrm{CuAg}$ and NiAu alloys is discussed more thoroughly in Sec. IV E.

(vii) In size-mismatched systems ( $\mathrm{Cu}-\mathrm{Ag}, \mathrm{Cu}-\mathrm{Au}$, and $\mathrm{Ni}-\mathrm{Au}) \Delta H_{\mathrm{SL}}(n, \widehat{G})$ exhibit the same order as the constituent strain $\Delta E_{\mathrm{CS}}^{\mathrm{eq}}(x, \widehat{G})$, i.e., $\Delta H_{\mathrm{SL}}(n,[001])$ is lowest and $\Delta H_{\mathrm{SL}}(n,[111])$ is highest. It suggests that low constituent strain stabilizes even short-period superlattices.

\section{Comparison of the trends in stability of metal and semiconductor superlattices}

Growth of semiconductor superlattices is a more mature are than than growth of metal superlattices, and much more data are available at present. Thus, it is of interest our results in Figs. 7 and 8 for metals with analogous results for semiconductors. ${ }^{72,73}$

Lattice-mismatched semiconductors generally have $\Delta H_{\mathrm{mix}}^{\text {bulk }}(x) \geq 0$ and $\Delta H_{\mathrm{SL}}^{\mathrm{bulk}} \geq 0$. Thus, they resemble $\mathrm{Ni}-\mathrm{Au}$ and $\mathrm{Cu}-\mathrm{Ag}$ rather than the compoundforming system $\mathrm{Cu}-\mathrm{Au}$. LDA calculations reveal that $\Delta H_{\mathrm{SL}}^{\mathrm{bulk}}(n, \widehat{G})$ for $G=\langle 111\rangle$ and $G=\langle 001\rangle$ look exactly like in $\mathrm{Cu}-\mathrm{Ag}$ or $\mathrm{Ni}-\mathrm{Au}$ : the energy decreases as the period $n$ increases, and the interfacial energies are mostly positive. However, in the $\langle 110\rangle$ and $\langle 201\rangle$ directions, the interfacial energies are negative, and $\Delta H_{\mathrm{SL}}^{\mathrm{bulk}}(n, \widehat{G})$ 


\section{Calculated Bulk and Epitaxial Energetics in Cu-Ag and Ni-Au}

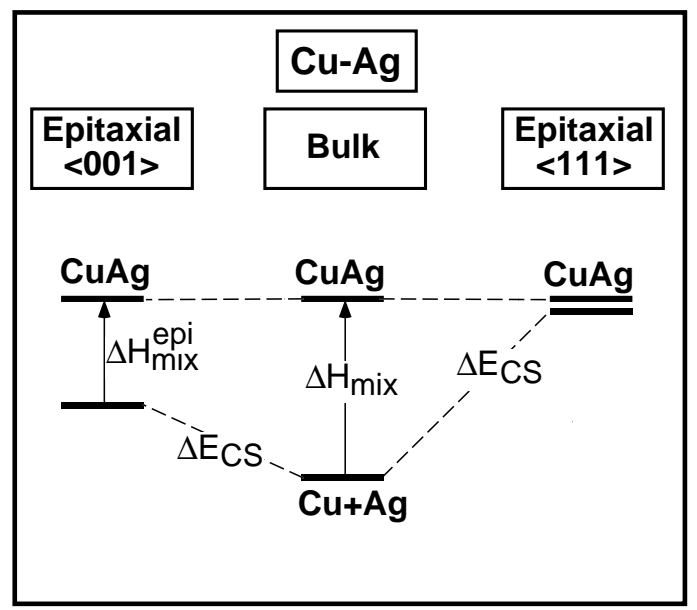

\section{CuAg on (111): Stable, but not at zero temperature}

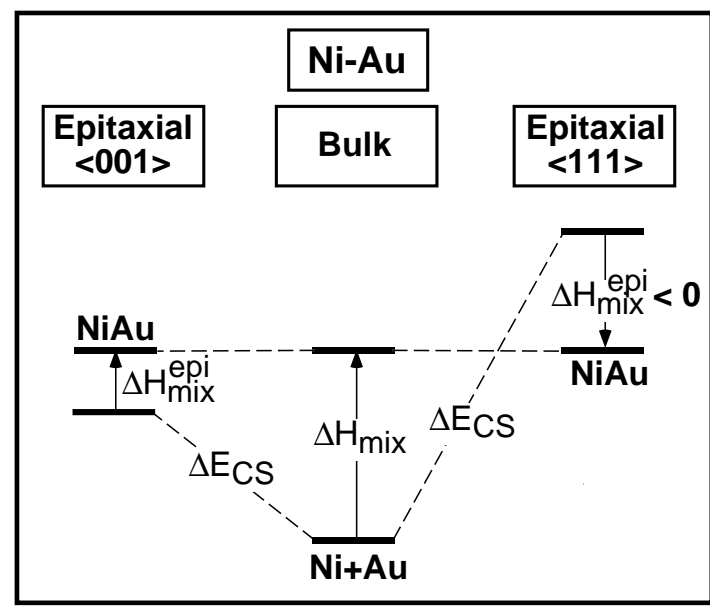

NiAu on (111): Stable!

FIG. 9. Mixing enthalpies $\Delta H_{\text {mix }}$ (in meV/atom) for bulk and epitaxial equiatomic Cu-Ag and Ni-Au alloys. All epitaxial calculations assume that the substrate is lattice matched to the random alloy. $\Delta E_{\mathrm{CS}}$ is the sum of epitaxial strain energies of pure elements [see Eq. (11)].

increases with $n$, like in $\mathrm{Ni}-\mathrm{Au}$ and $\mathrm{Cu}-\mathrm{Au}$. Hence, semiconductor superlattices behave generically as $\mathrm{Ni}-\mathrm{Au}$ superlattices. However, short-period 〈201〉 semiconductor superlattices (e.g., the chalcopyrite structure, corresponding to $n=2$ ) have a lower energy than the random alloy, while in $\mathrm{Ni}-\mathrm{Au}$ it is the $\langle 001\rangle$ short-period superlattices that have lower energies than the random alloy. Hence, while the Ni-Au random alloy can lower its energy by developing $\langle 001\rangle$ ordering, semiconductor random alloys can lower their energy by developing $\langle 201\rangle$ ordering. Both in Ni-Au and semiconductor alloys, the ultimate ground state is incoherent phase separation.

\section{E. Epitaxial growth and surface intermixing}

Recent experimental studies ${ }^{31,36}$ have grown epitaxial films of noble metal alloys which are immiscible in the bulk form. For instance, Stevens and Hwang ${ }^{36}$ have grown $\mathrm{Cu}-\mathrm{Ag}$ alloys on a $\mathrm{Ru}(0001)$ substrate, demonstrating that $\mathrm{Cu}$ and $\mathrm{Ag}$ are miscible at $T=823 \mathrm{~K}$, despite the fact that in bulk, $\mathrm{Cu}$ and $\mathrm{Ag}$ are strongly immiscible at this temperature and composition. It has also been observed that $\mathrm{Au}$ deposited on $\mathrm{Ni}(110)$ surface replaces it in the first surface layer forming a surface $\mathrm{Ni}-\mathrm{Au}$ alloy, ${ }^{31}$ although $\mathrm{Au}$ is completely insoluble in bulk Ni. In what follows we show that the stabilization of epitaxial solid solutions from bulk-immiscible con- stituents can be explained by the additional destabilization of the constituents due to the epitaxial constraint. Indeed, Eq. (11) shows that the epitaxial mixing enthalpy $\delta H_{\text {mix }}^{\text {epi }}$ may be considerably lower than the bulk mixing enthalpy $\Delta H_{\text {mix }}^{\text {bulk }}$ if the sum of the constituent strain energies on the right hand side is large.

Figure 9 shows the results for the epitaxial stabilization of equiatomic NiAu and $\mathrm{CuAg}$ alloys, assuming that the substrate is lattice matched to the disordered alloy.

(i) Disordered $\mathrm{CuAg}$ and NiAu alloys have large positive bulk mixing enthalpies $\Delta H_{\mathrm{mix}}^{\mathrm{bulk}}$, in agreement with the observed bulk immiscibility.

(ii) Epitaxy destabilizes the constituents, and hence stabilizes the epitaxial alloy in all cases. This effect is much larger for the elastically hard direction $\langle 111\rangle$ than for the soft $\langle 001\rangle$ direction.

(iii) The epitaxial mixing enthalpy $\delta H_{\mathrm{mix}}^{\mathrm{epi}}$ for $\langle 111\rangle$ becomes negative in $\mathrm{Ni}-\mathrm{Au}$, showing that the solid solution is energetically favored over the epitaxially phase separated state. In $\mathrm{CuAg}, \delta H_{\text {mix }}^{\text {epi }}$ is still positive and these alloys are unstable under epitaxial conditions at $T=0 \mathrm{~K}$.

(iv) Epitaxial conditions lead to a significantly enhanced miscibility since $\delta H_{\mathrm{mix}}^{\mathrm{epi}} \leq \delta H_{\mathrm{mix}}^{\mathrm{bulk}}$. A simple mean-field estimate of the miscibility gap temperature for $\mathrm{CuAg}$ grown on a nearly lattice-matched $\mathrm{Ru}(0001)$ substrate [equivalent to a fcc(111) substrate] gives $T_{\mathrm{MG}}=$ $2 \Delta H_{\mathrm{mix}}^{\mathrm{epi}}=150 \mathrm{~K}$. Thus, for (111)-epitaxy at the temperature $(823 \mathrm{~K})$ of Steven's and Hwang's experiment, 
our calculations predict complete solubility of $\mathrm{Cu}-\mathrm{Ag}$, as observed.

(v) The epitaxial stabilization is strongly dependent on the substrate orientation. A bigger effect can be observed for elastically hard directions, e.g., $\langle 111\rangle$ and $\langle 110\rangle$ for noble metal alloys.

\section{SUMMARY}

We have investigated the effects of anharmonic strain on the stability of epitaxial films, superlattices and epitaxially grown disordered alloys. We find that anharmonic epitaxial strain produces certain qualitative and quantitative features absent in the harmonic theory. In particular,

(i) Epitaxial softening functions $q\left(a_{s}, \widehat{G}\right)$ are strongly dependent on the substrate lattice constant $a_{s}$, while they are constants in the harmonic theory. For instance, as a consequence of the small fcc/bcc and fcc/bct energy difference, biaxially expanded $\mathrm{Cu}$ and Ni show drastic softening of $q\left(a_{s},[001]\right)$. Furthermore, biaxially compressed $\mathrm{Cu}, \mathrm{Ag}$, and $\mathrm{Au}$ have low values of $q\left(a_{s}, \widehat{G}\right)$ along directions $\langle 201\rangle$ and $\langle 110\rangle$ with relatively loose packing of atoms in the epitaxial planes.

(ii) The dependence of $q\left(a_{s}, \widehat{G}\right)$ on the direction $\widehat{G}$ can differ from harmonic predictions. For instance, $\langle 110\rangle$ is the hardest direction in biaxially expanded $\mathrm{Cu}$ and $\mathrm{Ni}$, and $\langle 201\rangle$ is the softest in biaxially compressed $\mathrm{Cu}, \mathrm{Ag}$ and Au. The harmonic formula always predicts either $\langle 111\rangle$ as the hardest and $\langle 001\rangle$ as the softest direction, or vice versa.

(iii) The strain energy of infinite coherent superlattices exhibits marked anomalies associated with the anharmonic behavior of constituent $q\left(a_{s}, \widehat{G}\right)$. The sizemismatched systems $\mathrm{Cu}-\mathrm{Ag}, \mathrm{Cu}-\mathrm{Au}$ and $\mathrm{Ni}-\mathrm{Au}$ exhibit very low constituent strain for Ag- and Au-rich $\langle 001\rangle$ superlattices, since $\langle 001\rangle$ is the easy direction for biaxial expansion of $\mathrm{Cu}$ and Ni. Similarly, 〈201〉 superlattices with small Ag or Au content have low coherency strain energies because this is the easy deformation direction for biaxially compressed Ag and Au. The in-plane lattice parameter $a_{\mathrm{SL}}$ of long-period $\langle 001\rangle$ superlattices suffers a discontinuous jump around $x \approx 0.2$, and other directions show considerable deviations from linear behaviour.

(iv) These elastic anomalies are less pronounced in short-period superlattices, although they contribute to the structural stability of $\langle 001\rangle$ superlattices. Shortperiod bulk superlattices are stable in $\mathrm{Ag}-\mathrm{Au}$ and $\mathrm{Cu}-\mathrm{Au}$ due to negative interfacial energies. Ag-Au and $\mathrm{Ni}-\mathrm{Au}$ have positive interfacial energies, leading to superlattice formation being energetically unfavorable with respect to phase separation. The interaction energy between interfaces in $\mathrm{Ni}-\mathrm{Au}$ is so strong that short-period $(n \propto 2)$ superlattices along $\langle 110\rangle$ are more stable than the longperiod superlattices with fewer interfaces. (v) Epitaxially grown disordered alloys can be stabilized even if the system phase separates in bulk form. This effect is caused by additional destabilization of the phase separated state due to the epitaxial constraint on the constituents, requiring them to be coherent with the substrate. The stabilization is more pronounced for elastically hard directions with high values of $q\left(a_{s}, \widehat{G}\right)$, e.g. $\langle 111\rangle$. For instance, we find that even though $\mathrm{Ni}-\mathrm{Au}$ and $\mathrm{Cu}-\mathrm{Ag}$ phase separate in the bulk $\left(\Delta H_{\text {mix }}^{\text {bulk }}(x)>0\right)$, equiatomic $\mathrm{Ni}_{0.5} \mathrm{Au}_{0.5}$ alloys are miscible when grown on a lattice-matched (111) substrate, while $\mathrm{Cu}_{0.5} \mathrm{Ag}_{0.5}$ on a (111) substrate is immiscible at $T=0 \mathrm{~K}$ but miscible at $T>150 \mathrm{~K}$. Neither $\mathrm{Ni}_{0.5} \mathrm{Au}_{0.5}$ nor $\mathrm{Cu}_{0.5} \mathrm{Ag}_{0.5}$ are miscible when grown on a lattice-matched (001) substrate, corresponding to a low energy penalty on the phase separated constituents.

\section{ACKNOWLEDGMENTS}

This work has been supported by the Office of Energy Research, Basic Energy Sciences, Materials Science Division, U.S. Department of Energy, under contract DEAC36-83CH10093.

${ }^{1}$ D. G. O'Neill and J. E. Houston, Phys. Rev. B 42, 2792 (1990).

2 M. A. Mueller, E. S. Hirschorn, T. Miller, and T.-C. Chiang, Phys. Rev. B 43, 11825 (1991).

${ }^{3}$ H. Brune, H. Röder, C. Boragno, and K. Kern, Phys. Rev. B 49, 2997 (1994).

${ }^{4}$ C. Günther, J. Vrijmoeth, R. Q. Hwang, and R. J. Behm, Phys. Rev. Lett. 74, 754 (1995).

${ }^{5}$ R. Q. Hwang, J. C. Hamilton, J. L. Stevens, and S. M. Foiles, Phys. Rev. Lett. 75, 4242 (1995).

${ }^{6}$ G. O. Pötschke and R. J. Behm, Phys. Rev. B 44, 1442 (1991).

${ }^{7}$ G. Vidali and H. Zeng, Appl. Surf. Sci. 92, 11 (1996).

${ }^{8}$ R. Ramirez, A. Rahman, and I. K. Schuller, Phys. Rev. B 30, 6208 (1984).

${ }^{9}$ E. Bauer and J. H. van der Merwe, Phys. Rev. B 33, 3657 (1986).

10 J. H. van der Merwe, CRC Critical Reviews in Solid State and Materials Sciences 17, 187 (1991).

${ }^{11}$ F. Gautier and D. Stoeffler, Surf. Sci. 249, 265 (1991).

12 C. Mottet, G. Treglia, and B. Legrand, Phys. Rev. B 46, 16018 (1992).

13 J. C. Hamilton and S. M. Foiles, Phys. Rev. Lett. 75, 882 (1995).

${ }^{14}$ Z. W. Lu, B. M. Klein, and A. Zunger, Superlattices and Microstructures 18, 161 (1995).

${ }^{15}$ W. P. Lowe, T. W. Barbee, Jr., T. H. Geballe, and D. B. McWhan, Phys. Rev. B 24, 6193 (1981). 
${ }^{16}$ I. Banerjee, Q. S. Yang, C. M. Falco, and I. K. Schuller, Phys. Rev. B 28, 5037 (1983).

${ }^{17}$ C. S. L. Chun, G.-G. Zheng, T. L. Vincent, and I. K. Schuller, Phys. Rev. B 29, 4915 (1984).

${ }^{18}$ A. Zunger, in Hanbook of Crystal Growth, Vol. 3, edited by D. T. J. Hurle (Elsevier, Amsterdam, 1994), p. 997, and references therein.

19 C. P. Wang, S. C. Wu, F. Jona, and P. Marcus, Phys. Rev. B 49, 17385 (1994).

${ }^{20}$ A. A. Saleh, V. Shutthanandan, and R. J. Smith, Phys. Rev. B 49, 4908 (1994).

${ }^{21}$ S. K. Kim, F. Jona, and P. M. Marcus, J. Phys. Condens. Matter. 8, 25 (1996).

${ }^{22}$ H. Wormeester, E. Hüger, and E. Bauer, Phys. Rev. Lett. 77, 1540 (1996).

${ }^{23}$ E. G. McRae and R. A. Malic, Surf. Sci. 177, 53 (1986).

${ }^{24}$ Y. Liu and P. Wynblatt, Surf. Sci. Lett. 241, L21 (1991).

${ }^{25}$ C. T. Chan, K. P. Bohnen, and K. M. Ho, Phys. Rev. Lett. 69, 1672 (1992).

${ }^{26}$ S. Rousset, S. Chiang, D. E. Fowler, and D. D. Chambliss, Phys. Rev. Lett. 69, 3200 (1992).

${ }^{27}$ D. D. Chambliss and S. Chiang, Surf. Sci. Lett. 264, L187 (1992).

${ }^{28}$ D. D. Chambliss, R. J. Wilson, and S. Chiang, J. Vac. Sci. Technol. A 10, 1992 (1993).

${ }^{29}$ H. Röder, R. Schuster, H. Brune, and K. Kern, Phys. Rev. Lett. 71, 2086 (1993).

${ }^{30}$ Y.-R. Tzeng, H.-T. Wu, K.-D. Shiang, and T. T. Tsong, Phys. Rev. B 48, 5549 (1993).

${ }^{31}$ L. Pleth Nielsen, F. Besenbacher, I. Stensgaard, E. Lægsgaard, C. Engdahl, P. Stoltze, K. W. Jacobsen, and J. K. Nørskov, Phys. Rev. Lett. 71, 754 (1993).

${ }^{32}$ L. Pleth Nielsen, I. Stensgaard, E. E. Lægsgaard, and F. Besenbacher, Surf. Sci. 307-309, 544 (1994).

${ }^{33}$ D. O. Boerma, G. Dorenbos, G. H. Wheatley, and T. M. Buck, Surf. Sci. 307-309, 674 (1994).

${ }^{34}$ E. I. Altman and R. J. Colton, Surf. Sci. Lett. 304, L400 (1994).

35 C. Nagl, M. Pinzolits, M. Schmid, and P. Varga, Phys. Rev. B 52, 16796 (1995).

36 J. L. Stevens and R. Q. Hwang, Phys. Rev. Lett. 74, 2078 (1995).

37 A. K. Schmid, J. C. Hamilton, N. C. Bartelt, and R. Q. Hwang, Phys. Rev. Lett. 77, 2977 (1996).

${ }^{38}$ D. L. Adams, Appl. Phys. 62, 123 (1996).

${ }^{39}$ W. C. Johnson and C. S. Chiang, J. Appl. Phys. 64, 1155 (1988).

${ }^{40}$ G. Bozzolo, R. Ibañez-Meier, and J. Ferrante, Phys. Rev. B 51, 7207 (1995).

${ }^{41}$ J. Tersoff, Phys. Rev. Lett. 74, 434 (1995).

${ }^{42}$ A. Christensen, A. V. Ruban, P. Stoltze, K. W. Jacobsen, H. L. Skriver, and J. K. Nørskov, Phys. Rev. B 56, 5822 (1997).

43 J. Hornstra and W. J. Bartels, J. Crystal Growth 44, 513 (1978).

${ }^{44}$ K. Yang, T. Anan, and L. J. Schowalter, Appl. Phys. Lett. 65, 2789 (1994).

${ }^{45}$ P. M. Marcus and F. Jona, Phys. Rev. B 51, 5263 (1995).

${ }^{46}$ D. J. Bottomley and P. Fons, J. of Crystal Growth 160, 406 (1996).
47 J. W. Cahn, Acta Met. 9, 795 (1961); ibid. 10, 179 (1962).

${ }^{48}$ F. C. Larché and J. W. Cahn, Acta Met. 33, 331 (1985); J. Appl. Phys. 62, 1232 (1987), and references therein.

${ }^{49}$ B. de Cremoux, J. Phys. Colloq. 43, C5-19 (1982).

${ }^{50}$ C. P. Flynn, Phys. Rev. Lett. 57, 599 (1986).

51 J. B. Stringfellow, J. Appl. Phys. 43, 3455 (1972); J. Electronic Mater. 11, 903 (1982); J. Cryst. Growth 65, 454 (1983).

${ }^{52}$ C. S. Chiang and W. C. Johnson, J. Mater. Res. 4, 678 (1989).

53 D. M. Wood and A. Zunger, Phys. Rev. Lett. 61, 1501 (1988); Phys. Rev. B 38, 12756 (1988); ibid. 40, 4062 (1989).

${ }^{54}$ D. M. Wood, J. Vac. Sci. Technol. B 10, 1675 (1992), and references therein.

${ }^{55}$ I. P. Ipatova, V. A. Shchukin, V. G. Malyshkin, A. Yu. Maslov, and E Anastassakis, Solid State Commun. 78, 19 (1991); V. G. Malyshkin and V. A. Shchukin, Semiconductors 27, 1062 (1993); I. P. Ipatova, V. G. Malyshkin, and V. A. Shchukin, J. Appl. Phys. 74, 7198 (1993).

${ }^{56}$ D. B. Laks, L. G. Ferreira, S. Froyen, and A. Zunger, Phys. Rev. B 46, 12587 (1992).

${ }^{57}$ A. Zunger, in NATO ASI on Statics and Dynamics of Alloy Phase Transformations, (Plenum Press, New York, 1994), p. 361.

${ }^{58}$ P. Hohenberg and W. Kohn, Phys. Rev. 136, 864 (1964); W. Kohn and L. J. Sham, Phys. Rev. A 136, 1133 (1965).

${ }^{59}$ S.-H. Wei and H. Krakauer, Phys. Rev. Lett. 55, 1200 (1985), and references therein. We used the Wigner exchange-correlation functional [E. Wigner, Phys. Rev. 46, 1002 (1934)] and equivalent $\mathbf{k}$ point meshes [S. Froyen, Phys. Rev. B 39, 3168 (1989)] corresponding to 60 points in the irreducible part of the fcc Brillouin zone. (Tests with a $16 \times 16 \times 16$ special points mesh showed negligible differences in the calculated $q\left(a_{s}, \widehat{G}\right)$.) The muffin-tin radii were $R_{\mathrm{Au}}=2.4 a_{0}, R_{\mathrm{Ag}}=R_{\mathrm{Cu}}=R_{\mathrm{Ni}}=2.2 a_{0}$, and the basis set was defined by $R K_{\max }=9$. Valence states were calculated semirelativistically (no spin-orbit) [A. H. MacDonald, W. E. Pickett, and D. D. Koelling, J. Phys. C 13, 2675 (1980); W. E. Pickett, A. J. Freeman, and D. D. Koelling, Phys. Rev. B 23, 1266 (1981)], neglecting electron spin polarization effects. Further details can be found in Ref. 60 .

${ }^{60}$ V. Ozolinšs, C. Wolverton, and A. Zunger, to appear in Phys. Rev. B (1998).

${ }^{61}$ C. Wolverton and A. Zunger, Comp. Mater. Sci. 8, 107 (1997); C. Wolverton, V. Ozoliņš, and A. Zunger, to appear in Phys. Rev. B (1998).

62 S.-H. Wei and H. Krakauer, Phys. Rev. Lett. 55, 1200 (1985), and references therein.

${ }^{63}$ E. C. Bain, Trans. Am. Inst. Min. Metall. Eng. 70, 25 (1924).

${ }^{64}$ T. Kraft, P. M. Marcus, M. Methfessel, and M. Scheffler, Phys. Rev. B 48, 5886 (1993).

${ }^{65}$ P. Alippi, P. M. Marcus, and M. Scheffler, Phys. Rev. Lett 78, 3892 (1997).

${ }^{66}$ M. Sob, L. G. Wang, and V. Vitek, Comp. Mater. Sci. 8, 100 (1997).

${ }^{67}$ Indeed, since $E^{\prime}(\gamma)<0$ for $\gamma \ll \gamma_{\mathrm{bcc}}$ and $E^{\prime}(\gamma)>0$ for $\gamma \gg \gamma_{\mathrm{fcc}}$, the derivative $E^{\prime}(\gamma)$ must pass through zero an odd number of times, proving that in addition to the 
two points of cubic symmetry there must exist a third extremal point corresponding to the bct structure. The implicit assumption that there are no inflection points does not change the essence of the discussion.

${ }^{68}$ P. J. Craievich, M. Weinert, J. M. Sanchez, and R. E. Watson, Phys. Rev. Lett. 723076 (1994).

${ }^{69}$ P. J. Craievich, J. M. Sanchez, R. E. Watson, and M. Weinert, Phys. Rev. B 55787 (1997).

${ }^{70}$ A. Fernández Guillermet, V. Ozoliņš, G. Grimvall, and M. Körling, Phys. Rev. B 51, 10364 (1995).

${ }^{71}$ S.-H. Wei, private communication. In GaP, $q\left(a_{s},[001]\right)$ increases from 0.364 at $a_{s}=5.618 \AA$ to 0.383 at $a_{s}=5.888 \AA$, and $q\left(a_{s},[111]\right)$ increases from 0.534 at $a_{s}=5.618 \AA$ Ato 0.559 at $a_{s}=5.888 \AA$.

${ }^{72}$ R. G. Dandrea, J. E. Bernard, S.-H. Wei, and A. Zunger, Phys. Rev. Lett. 64, 36 (1990).

${ }^{73}$ S.-H. Wei and A. Zunger, Phys. Rev. Lett. 61, 1505 (1988). 